\title{
Ischemic Postconditioning Alleviates Cerebral Ischemia-Reperfusion Injury Through Activating Autophagy During Early Reperfusion in Rats
}

\author{
Yameng Sun ${ }^{1} \cdot$ Ting Zhang ${ }^{1} \cdot$ Yan Zhang ${ }^{2} \cdot$ Jinfeng $\mathrm{Li}^{2} \cdot$ Lei Jin $^{2} \cdot$ Yinyi Sun $^{1} \cdot \mathrm{Nan} \mathrm{Shi}^{2} \cdot \mathrm{Kangyong} \mathrm{Liu}^{2} \cdot \mathrm{Xiaojiang} \mathrm{Sun}^{1}$
}

Received: 4 August 2017 / Revised: 25 May 2018 / Accepted: 20 July 2018 / Published online: 25 July 2018

(C) The Author(s) 2018

\begin{abstract}
This study aimed to investigate whether ischemic postconditioning (IpostC) alleviates cerebral ischemia/reperfusion (I/R) injury involved in autophagy. Adult Sprague-Dawley rats were divided into five groups: sham (sham surgery), I/R (middle cerebral artery occlusion [MCAO] for $100 \mathrm{~min}$, then reperfusion), IpostC (MCAO for $100 \mathrm{~min}$, reperfusion for $10 \mathrm{~min}$, $\mathrm{MCAO}$ for $10 \mathrm{~min}$, then reperfusion), IpostC+3MA (3-methyladenine, an autophagy inhibitor, administered 30 min before first reperfusion), and IpostC+Veh (vehicle control for IpostC+3MA group). Infarct volume was measured using cresyl violet staining. Autophagy-related proteins were detected by western blot and immunohistochemistry. Autophagosomes, autophagolysosomes, and mitochondrial damage were identified by transmission electron microscopy. Cortical cell apoptosis was detected by the TUNEL assay. Neurologic function was assessed using the modified Neurologic Severity Score. IpostC improved neurological function and reduced infarct volume after $\mathrm{I} / \mathrm{R}(P<0.05)$. These effects of IpostC were inhibited by $3 \mathrm{MA}(P<0.05)$. Autophagosome formation was increased in the $\mathrm{I} / \mathrm{R}$ and IpostC + Veh groups $(P<0.05)$, but not in the IpostC+3MA group. The I/R group showed enhanced LC3-II/LC3-I ratio, p62, and Cathepsin B levels and decreased LAMP-2 level (all $P<0.05$ vs. sham), indicating dysfunction of autophagic clearance. IpostC reduced p62 and Cathepsin B levels and increased the LC3-II/LC3-I ratio, and nuclear translocation of transcription factor EB (all $P<0.05$ ); these effects of IpostC were reversed by $3 \mathrm{MA}$, suggesting IpostC enhanced autophagic flux. Furthermore, IpostC attenuated I/R-induced mitochondrial translocation of Bax and mitochondrial cytochrome-c release (all $P<0.05$ ); 3MA inhibited these effects of IpostC $(P<0.05)$. In conclusion, IpostC may alleviate cerebral $\mathrm{I} / \mathrm{R}$ injury by activating autophagy during early reperfusion.
\end{abstract}

Keywords Ischemic postconditioning $\cdot$ Autophagy flux $\cdot$ Cerebral ischemia-reperfusion injury $\cdot$ Mitochondrial dysfunction

Electronic supplementary material The online version of this article (https://doi.org/10.1007/s11064-018-2599-3) contains supplementary material, which is available to authorized users.

Kangyong Liu

liukangyong5555@163.com

$\triangle$ Xiaojiang Sun sunxj155@sohu.com

1 Department of Neurology, Shanghai Sixth People's Hospital, Shanghai Jiao Tong University School of Medicine, Shanghai 200233, People's Republic of China

2 Department of Neurology, Shanghai University of Medicine and Health Sciences Affiliated Zhoupu Hospital, Shanghai 201318, People's Republic of China

\section{Introduction}

Stroke is an acute disorder with serious complications and high mortality caused by impaired blood supply to the brain. Stroke is the fifth leading cause of death and the first cause of disability in adults [1]. The annual incidence of stroke is 795,000 in the USA, with an average of one victim every $40 \mathrm{~s}$ [1]. In China, the age-standardized incidence and prevalence of stroke are $1115 / 100,000$ people and 247/100,000 person-years, with a mortality of $115 / 100,000$ person-years [2]. Ischemic stroke accounts for $60-80 \%$ of all cases [2]. Despite an estimated cost of $>70$ billion dollars per year, the treatment of stroke remains very limited and is mainly based on supportive therapies [1].

Restoration of blood supply during the reperfusion phase can induce ischemia-reperfusion (I/R) injury. Preconditioning is a brief subcritical ischemic episode that can mobilize intrinsic protective mechanisms against subsequent 
detrimental ischemia [3]. Since most cerebral ischemic events occur unpredictably, postconditioning (postC) could provide more therapeutic opportunities than preconditioning. Ischemic postC (IpostC) was first reported by Zhao et al. in 2006 [4]. IpostC is defined as a series of rapid intermittent interruptions of blood flow during reperfusion, and mobilizes the same protective mechanisms than preconditioning $[3,4]$. Following acute ischemia, IpostC was found to decrease the necrotic area by $80 \%$ and reduce the extent of apoptosis $[4,5]$.

Autophagy is a process by which intracellular proteins and organelles are delivered to lysosomes by autophagosomes for degradation and recycling. Three types of autophagy are currently recognized: macroautophagy, microautophagy, and chaperone-mediated autophagy (CMA) [6]. Several key proteins in the autophagic cascade have been identified. Among them, Beclin-1 is essential for recruitment of autophagic proteins [7]. Markers of autophagic activity include the ratio of LC3-I (cytosolic) to LC3-II (membranebound), which correlates with autophagosome formation [8], whereas p62 levels are inversely correlated with autophagic activity [9]. LAMP-2 is another important protein involved in autophagy [10]. LAMP-2 deficiency causes autophagic vacuole accumulation in various tissues, resulting in cardiomyopathy and Danon disease in mice [11]. Accordingly, a recent study showed that chronic brain hypoperfusion in rats led to inefficient lysosomal clearance, which was caused by downregulation of LAMP-2 [12]. Cathepsin B belongs to the lysosomal cysteine protease family; it is upregulated in many pathological conditions, including ischemia, and its inactivation attenuates the ischemic apoptotic injury and autophagy [13-15]. Finally, under stress inducing autophagy, the bHLH-leucine zipper transcription factor EB (TFEB) translocates from the cytoplasm to the nucleus to regulate genes involved in autophagosome formation, autophagosome-lysosome fusion, and lysosomal exocytosis [16-19].

Several IpostC strategies have been demonstrated to activate autophagy in the ischemic brain [20-28]. Nevertheless, it remains unclear whether autophagy participates in the neuroprotective effects of IpostC in cerebral I/R [29-31]. Indeed, the roles of autophagy in IpostC are controversial. Some studies have reported that autophagy eliminates the neuroprotective effects of IpostC [22, 27, 32], whereas others have found that autophagy activation mimics IpostC-mediated neuroprotection [20,26]. The apparent contradiction might be attributable to the complex nature of autophagy with regard to cargo selection [33]. In ischemic brains, the clearance of impaired mitochondria by autophagy is beneficial for neuronal survival, whereas nonselective autophagy is detrimental [31, 34]. Additional research is needed to better characterize the role of autophagy in the reperfusion phase after ischemia and its contribution to the final fate of neurons. Therefore, in the present study, we hypothesized that autophagy is a key process during IpostC and protects the ischemic brain.

\section{Methods and Materials}

\section{Animals}

All experimental procedures were approved by the Institutional Animal Care and Use Committee of Shanghai Jiao Tong University, Shanghai, China. Adult male Sprague-Dawley rats (9-10 weeks old, weighing 260-320 g) were obtained from the Shanghai Laboratory Animal Center, Shanghai, China. The rats were housed in individual cages at a constant room temperature with a 12-h light/dark cycle and were given free access to water and food. Every effort was made to minimize the number of rats used and animal suffering.

\section{Rat Model of Transient Middle Cerebral Artery Occlusion (MCAO) and Administration of Drugs}

Transient focal cerebral ischemia and focal IpostC were carried out as previously described [35]. All surgical procedures were performed under an operating stereomicroscope, and body temperature (monitored by a rectal probe) was maintained at $37 \pm 0.5^{\circ} \mathrm{C}$ with a thermostatically controlled heating pad. Anesthesia was induced in a glass chamber using 4\% isoflurane (mixed with $70 \% \mathrm{~N}_{2} \mathrm{O}$ and $30 \% \mathrm{O}_{2}$ ) and maintained with a facemask using $2 \%$ isoflurane. A midline incision was made on the ventral neck surface, and the left common carotid artery (CCA), external carotid artery (ECA), and internal carotid artery (ICA) were isolated. A silicone-coated, round-tipped 4-0 monofilament nylon suture (Covidien, Mansfield, MA, USA) was gently introduced from the left CCA into the ICA through the stump of the ECA and advanced $20 \pm 1 \mathrm{~mm}$ into the circle of Willis to occlude the branch point of the left middle cerebral artery (MCA). Reperfusion was performed 100 min after MCAO by withdrawal of the suture. Laser Doppler flowmetry (Moor Instruments, Devon, UK) was used to confirm ischemia in the area supplied by the left MCA. Animals without a cerebral blood flow reduction of at least $70 \%$ were excluded from the analysis, as were animals that died after induction of ischemia. The mortality in our study was less than 5\%.

The autophagy inhibitor 3-methyladenine (3MA; M9281, Sigma-Aldrich, St. Louis, MO, USA) was administered by intracerebral ventricle (icv) injection; the dose (400 nM) was chosen according to a previous study [22]. Control rats received an equal volume of sterile saline. The anesthetized rat was placed on a stereotaxic apparatus, and 3MA or sterile saline was infused by a microinjector $(1 \mu \mathrm{L} / \mathrm{min})$ into the ventricular space ipsilateral to the ischemic region 
(-0.92 $\mathrm{mm}$ anteroposterior, $1.5 \mathrm{~mm}$ mediolateral, and $3.5 \mathrm{~mm}$ dorsoventral to the bregma). Before each treatment, $3 \mathrm{MA}$ was freshly prepared in normal saline (final concentration, $100 \mathrm{nmol} / \mu \mathrm{L}$ ) by heating the solution to $60-70{ }^{\circ} \mathrm{C}$.

\section{Experimental Design}

The animals were randomly assigned to five groups: sham group (surgical procedure undertaken but without MCAO); $\mathrm{I} / \mathrm{R}$ group (MCAO for $100 \mathrm{~min}$ followed by reperfusion for $1,6,12,24$, or $48 \mathrm{~h}$ ); IpostC group (MCAO for $100 \mathrm{~min}$, reperfusion for $10 \mathrm{~min}, \mathrm{MCAO}$ for a further $10 \mathrm{~min}$, then reperfusion for 1, 6, 12, 24, or $48 \mathrm{~h}$ ); IpostC+3MA group (MCAO for $100 \mathrm{~min}$, reperfusion for $10 \mathrm{~min}$ with $400 \mathrm{nM}$ of 3MA administered 30 min before reperfusion, MCAO for a further $10 \mathrm{~min}$, then reperfusion for $24 \mathrm{~h}$ ); and IpostC+Veh group (as for the IpostC+3MA group, but with an equal volume of sterile saline administered instead of 3MA). The experimental design and number of animals used are presented in Figure S1.

\section{Immunoblotting Analysis}

The rats were anesthetized with an intraperitoneal injection of $10 \%$ chloral hydrate $(350 \mathrm{mg} / \mathrm{kg}$ ) and euthanized (by decapitation) after $1,6,12,24$, or $48 \mathrm{~h}$ of reperfusion ( $\mathrm{n}=3$ rats/group/time point). The brains were removed on ice and the cerebral cortices ipsilateral to the ischemic area were dissected out rapidly and the tissues bordering the ischemic region were taken into RIPA lysis buffer (Millipore, Bedford, MA, USA) supplemented with $1 \mathrm{mmol} / \mathrm{L}$ phenylmethylsulfonyl fluoride (Thermo Fisher Scientific, Waltham, MA, USA) and a cocktail of protease inhibitors (Thermo Fisher Scientific). The tissues were sonicated and the protein concentration in the supernatant fraction was determined using a bicinchoninic acid assay (Thermo Fisher Scientific). The proteins $(40 \mu \mathrm{g})$ were separated by electrophoresis on 8 , 10 , or $12 \%$ sodium dodecyl sulfate polyacrylamide gels and transferred onto 0.45 - $\mu \mathrm{m}$ nitrocellulose membranes (Whatman, Piscataway, NJ, USA) or 0.2- $\mu$ m polyvinylidene fluoride membranes (HVPPEAC12, Millipore). The membranes were blocked in 5\% nonfat milk for $1 \mathrm{~h}$ and incubated overnight at $4{ }^{\circ} \mathrm{C}$ with primary antibodies against LC3 $(1: 1000$, PM036, MBL, Nagoya, Japan), beclin-1 (1:400, sc-11427, Santa Cruz Biotechnology, Santa Cruz, CA, USA), p62 (1:1000, PM045, MBL), Bax (1:1000, \#2772, Cell Signaling Technology, Danvers, MA, USA), cytochrome-c (1:1000, \#11940, Cell Signaling Technology), $\beta$-actin (1:1000, sc-47778, Santa Cruz Biotechnology, Santa Cruz, CA, USA), LAMP-2 (1:200, sc-34245, Santa Cruz Biotechnology, Santa Cruz, CA, USA), TFEB (1:200, sc-48784, Santa Cruz Biotechnology, Santa Cruz, CA, USA), cytochrome-c oxidase subunit IV (COX IV; 1:1000, \#4850, Cell Signaling
Technology), cathepsin B (1:200, sc-365558, Santa Cruz Biotechnology, Santa Cruz, CA, USA) and H3 (1:1000, \#9728, Cell Signaling Technology). The membranes were subsequently washed, incubated with horseradish peroxidase (HRP)-conjugated secondary antibodies for $1 \mathrm{~h}$ at room temperature and reacted with enhanced chemiluminescence substrate (Pierce, Rockford, IL, USA). The results were recorded using Quantity One imaging software (Bio-Rad, Hercules, CA, USA), and relative intensities were calculated using Gel-Pro Analyzer software (Media Cybernetics, Bethesda, MD, USA). For some experiments, subcellular fractionation was performed using a Nuclear and Cytoplasmic Protein Extraction Kit (p0027, Beyotime Biotechnology, Shanghai, China) $[36,37]$ and Tissue Mitochondria Isolation Kit (C3606, Beyotime Biotechnology) [38, 39].

\section{Transmission Electron Microscopy (TEM)}

Cortical tissues bordering the ischemic core area from rats sacrificed at $24 \mathrm{~h}$ after ischemia ( $\mathrm{n}=3$ rats/group) was fixed in $2.5 \%$ glutaraldehyde in $0.1 \mathrm{~mol} / \mathrm{L}$ phosphate-buffered saline (PBS, pH 7.4) and cut by a vibratome into $50-\mu \mathrm{m}$-thick sections. The sections were postfixed with $1 \%$ osmium tetroxide for $1 \mathrm{~h}$, dehydrated in a graded ethanol series (up to $100 \%$ ) followed by dry acetone, and then embedded in Durcupan ACM Fluka (Sigma Aldrich, St Louis, MO, USA). The sections were further cut with a Reichert ultramicrotome (Leica, Wetzlar, Germany) into ultrathin $(70 \mathrm{~nm})$ sections that were stained with uranyl acetate and lead citrate and visualized using a CM-120 electron microscope (Philips, Amsterdam, Netherlands) [40].

For quantitative analyses of the numbers of autophagosomes, autolysosomes, and mitochondria in each group, 20 fields from each of three rats were examined using a protocol described previously [40]. Twenty-five randomly selected TEM images per animal were captured at a final magnification of 20,000x, and the number of autophagosomes and autolysosomes in each captured field was counted by visual inspection using previously established criteria for identification [7]. Autophagosomes were defined as double-membrane structures with an internal density similar to that of surrounding cytosol, while autolysosomes were considered to have only one limiting membrane and contained cytoplasmic material/organelles at various stages of degradation. For mitochondrial analysis, at least 20 randomly selected fields from tissues bordering the ischemic core area per animal were captured at a final magnification of $20,000 \times$, and the number of mitochondria in each field from tissues surrounding the ischemic region was calculated by a technician blinded to the experimental design. The number of swollen mitochondria was also assessed. A mitochondrion was considered swollen when 5-6 times larger than normal mitochondria or in the presence of membrane rupture [41, 42]. 


\section{Immunohistochemistry}

Each animal ( $\mathrm{n}=3$ rats/group) was anesthetized $24 \mathrm{~h}$ after the surgical procedure and perfused transcardially with $4 \%$ paraformaldehyde in $0.1 \mathrm{~mol} / \mathrm{L} \mathrm{PBS} \mathrm{(pH} \mathrm{7.4).} \mathrm{The} \mathrm{brain} \mathrm{was}$ removed and postfixed for $24 \mathrm{~h}$ in $4 \%$ paraformaldehyde. Coronal sections bordering the ischemic core area were dehydrated in a graded ethanol series and then embedded in paraffin. Serial coronal sections $(5 \mu \mathrm{m})$ were cut with a microtome, dewaxed in xylene, and rehydrated in graded alcohol. After incubation in PBS containing 0.3\% Triton X-100 for $10 \mathrm{~min}$, the mounted tissue sections underwent microwave antigen retrieval. To quench endogenous peroxidase activity, the sections were incubated in $0.3 \% \mathrm{H}_{2} \mathrm{O}_{2}$ in methanol for $30 \mathrm{~min}$, rinsed in PBS, and blocked in $1 \%$ horse serum (Vector Laboratories, Burlingame, CA, USA) dissolved in PBS for $60 \mathrm{~min}$ at room temperature. The tissues were subsequently incubated overnight at $4{ }^{\circ} \mathrm{C}$ with primary antibodies against LC3 (1:1000, L7543, Sigma-Aldrich, St Louis, Mo, USA), beclin-1 (1:1000, \#3738, Cell Signaling Technology, Danvers, MA, USA), and p62 (1:1000, BMLPW9860, Enzo Life Sciences, Farmingdale, NY, USA). The sections were then incubated with biotinylated universal antibody (Vector Laboratories, Burlingame, CA, USA) for $60 \mathrm{~min}$ at room temperature, rinsed in PBS, incubated in Vectasta in $A B C$ reagent for 60 min and rinsed again in PBS. The reaction product was visualized using 3,3'-diaminobenzidine (Vector Laboratories, Burlingame, CA, USA) until the desired intensity of staining was obtained. The sections were finally dehydrated in graded alcohol and mounted in Eukitt (Electron Microscopy Sciences, Hatfield, PA, USA) mounting medium. For each rat, five consecutive sections spaced at $200 \mu \mathrm{m}$ were photographed for each of four regions of interest in the ipsilateral hemisphere, including the perifocal region in the cortex.

\section{Terminal Deoxynucleotidyl Transferase-Mediated dUTP Nick-end Labeling (TUNEL) Assay}

The sections (from $n=3$ rats/group, sacrificed at $24 \mathrm{~h}$ ) were permeabilized with $0.4 \%$ Triton X-100 for $10 \mathrm{~min}$ and incubated with $10 \%$ normal donkey serum for $1 \mathrm{~h}$ at room temperature followed by anti-NeuN antibody (1:200, MAB377, Millipore corp., Billerica, MA, USA) overnight at $4{ }^{\circ} \mathrm{C}$. The tissues were washed ( $0.1 \%$ Triton X-100 for 3-10 min), incubated with donkey anti-mouse Alexa Fluor 594-conjugated secondary antibody at room temperature for $2 \mathrm{~h}$, and then washed again (PBS for 3-10 min). TUNEL staining was performed using an in situ cell death detection kit (Roche Applied Science, Nonnenwald, Germany), in accordance with the manufacturer's instructions. The mounted sections were incubated for $2 \mathrm{~min}$ in a freshly prepared aqueous solution of $0.1 \%$ Triton $\mathrm{X}-100$ and $0.1 \%$ sodium citrate, immersed in $50 \mu \mathrm{L}$ of TUNEL mixture for $1 \mathrm{~h}$ at $37{ }^{\circ} \mathrm{C}$, rinsed in PBS, and mounted with FluorSave. For peroxidase revelation of TUNEL, sections were incubated for $2 \mathrm{~h}$ at room temperature with an anti-FITC antibody conjugated with biotin (Sigma-Aldrich, St Louis, MO, USA). The sections were viewed and analyzed using laser scanning confocal microscopy (LSCM, FV1000, Olympus, Tokyo, Japan) and a digital imaging software (FV10-ASW 1.5 Viewer). The number of NeuN-positive or TUNEL-positive neurons per 200- $\mu \mathrm{m}$ length of cortical tissue bordering the ischemic core area were counted in five sections per animal. Cell counts on each of the five sections were averaged to provide the mean value. The mean value was used for analysis.

\section{Neurobehavioral Assessments}

Neurologic function ( $\mathrm{n}=9$ rats/group) was assessed $24 \mathrm{~h}$ after reperfusion by an experimenter blinded to the animal grouping. A modified Neurologic Severity Score (mNSS) ranging $0-14$ was adopted. The mNSS assessment included raising the rat by the tail ( $0-3)$, walking on the floor $(0-3)$, beam balance tests $(0-6)$, and absence of reflexes $(0-2)$ [43].

\section{Measurements of Infarct Volume}

Infarct volume was determined using cresyl violet staining (from $\mathrm{n}=6$ rats/group, sacrificed at $24 \mathrm{~h}$ ). A series of $20-\mu \mathrm{m}$-thick coronal sections from the anterior commissure to the hippocampus was obtained, and the first of every 10 consecutive sections were mounted on slides (i.e. a distance between adjacent sections on the slides of $200 \mu \mathrm{m}$ ), for a total of 18-25 sections. The frozen sections were stained with cresyl violet (Sigma-Aldrich, St Louis, MO, USA), and the ischemic area within each section was delineated using an image analysis software (ImageJ, National Institutes of Health, Bethesda, MD, USA). The infarct volume between two adjacent sections was calculated using the formula: $1 / 3 \times \mathrm{h} \times[\mathrm{S} 1+\mathrm{S} 2+\sqrt{(\mathrm{S} 1 \times \mathrm{S} 2)}]$, where $\mathrm{S} 1$ and $\mathrm{S} 2$ are the infarct areas of the two sections. Infarct volume was derived from the sum of all infarct volumes between adjacent sections.

\section{Statistical Analysis}

Statistical analysis was carried out using Prism 5 software (GraphPad Software, San Diego, CA, USA). Data are presented as mean \pm standard deviation (SD) (for mNSS score and infarct volume) or mean \pm standard error of the mean (SEM) (for western blot and TEM). Comparisons among multiple groups were made using one-way (Figs. 2, 3, 4, $5,6,7$ ) or two-way (Fig. 1) analysis of variance (ANOVA) 
Fig. 1 Effects of ischemic postconditioning (IpostC) on time-dependent expression levels of LC3, p62, and Beclin 1 in the cerebral cortex following ischemia-reperfusion (I/R) injury. Rats were subjected to cerebral I/R injury with or without IpostC, and this was followed by reperfusion for 1 , $6,12,24$, or 48 h. LC3, p62, and Beclin 1 protein expression levels in cerebral cortex were determined by western blotting. a LC3 expression at different times after reperfusion in the I/R group. b LC3 expression at different times after reperfusion in the IpostC group. c p62 expression at different times after reperfusion in the I/R group. d p62 expression at different times after reperfusion in the IpostC group. e Beclin 1 expression at different times after reperfusion in the $\mathrm{I} / \mathrm{R}$ group. $\mathbf{f}$ Beclin 1 expression at different times after reperfusion in the IpostC group. Data presented as the mean \pm standard error of the mean (SEM) ( $\mathrm{n}=3$ each group). $* P<0.05$, $* * P<0.01, * * * P<0.001$ vs. sham group; ${ }^{\triangle} P<0.05$ vs. IpostC $24 \mathrm{~h} ;{ }^{\&} P>0.05$ vs. IpostC $24 \mathrm{~h}$
A

B
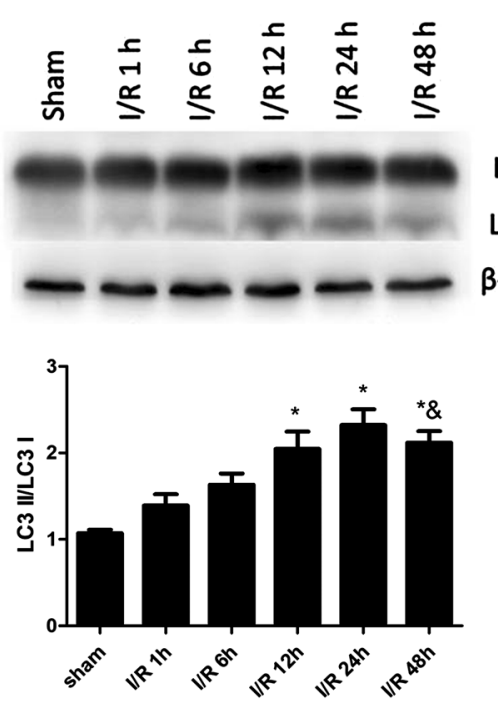

LC3-I

LC3-II

$\beta$-actin $-\infty-\infty$

C
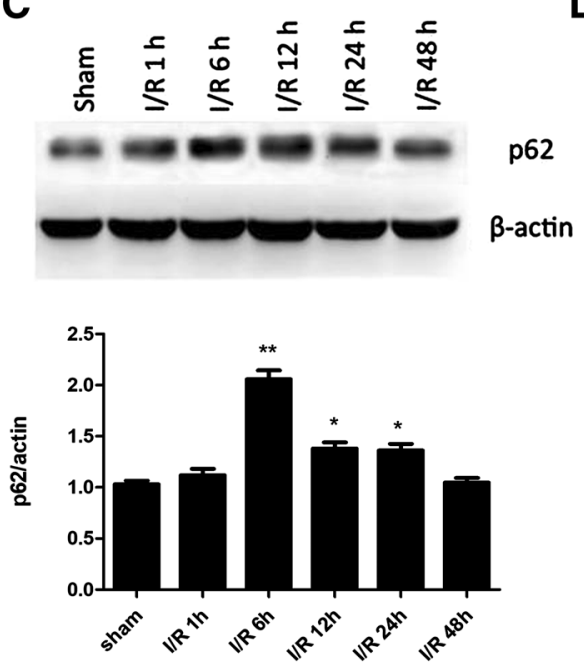

E
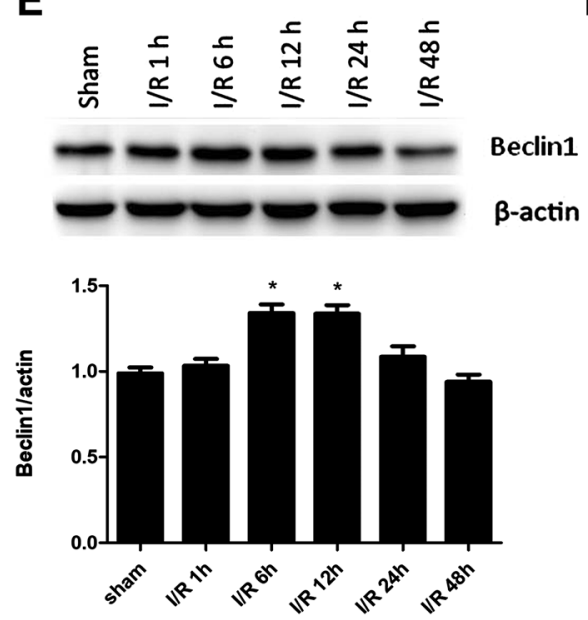
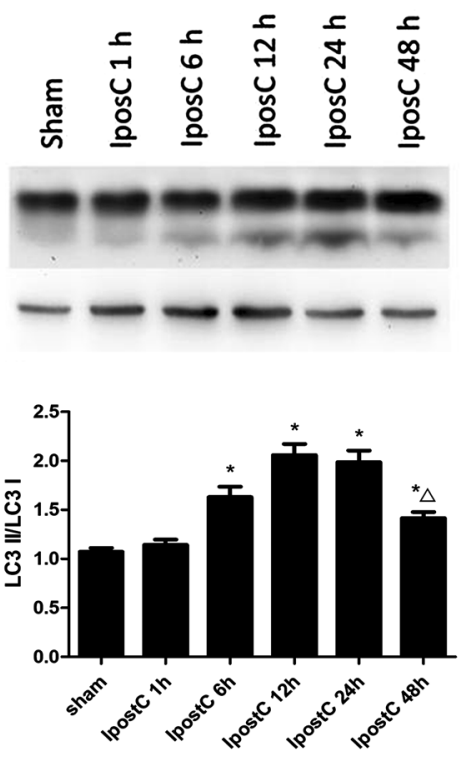

D
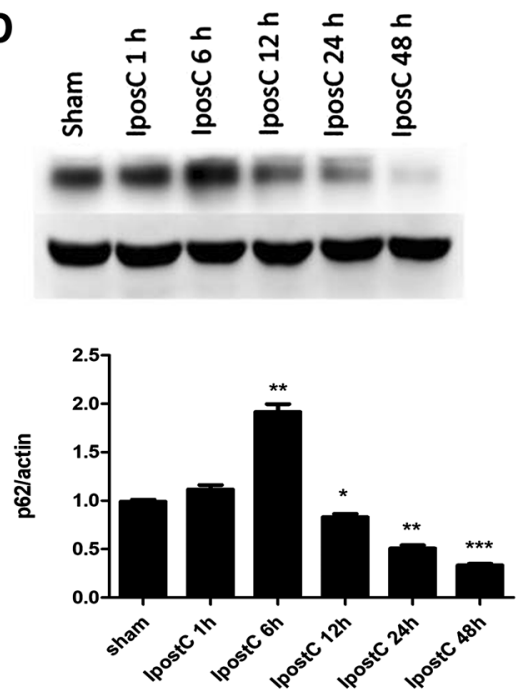

$\mathbf{F}$
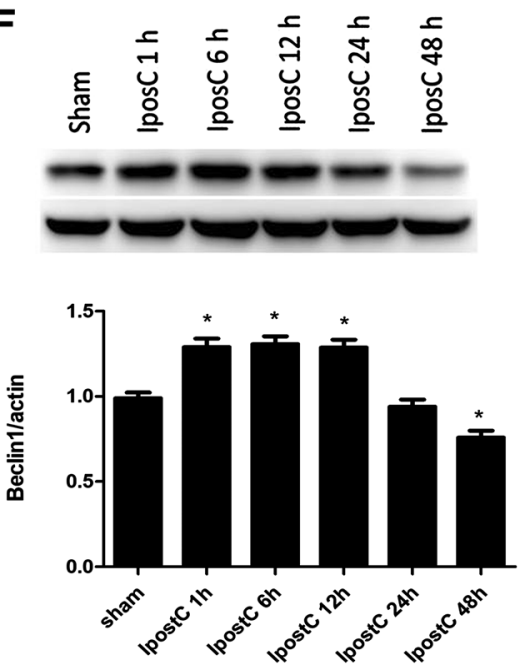


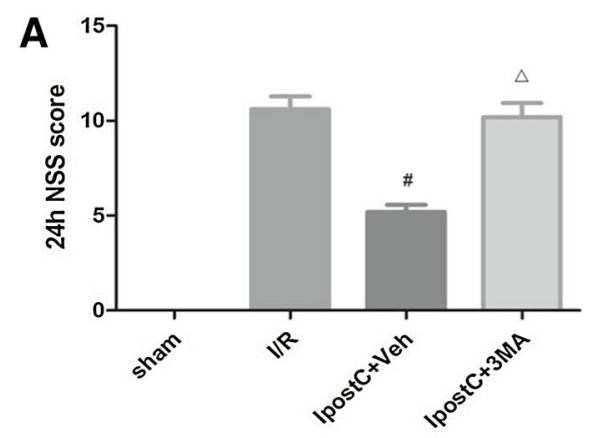

B
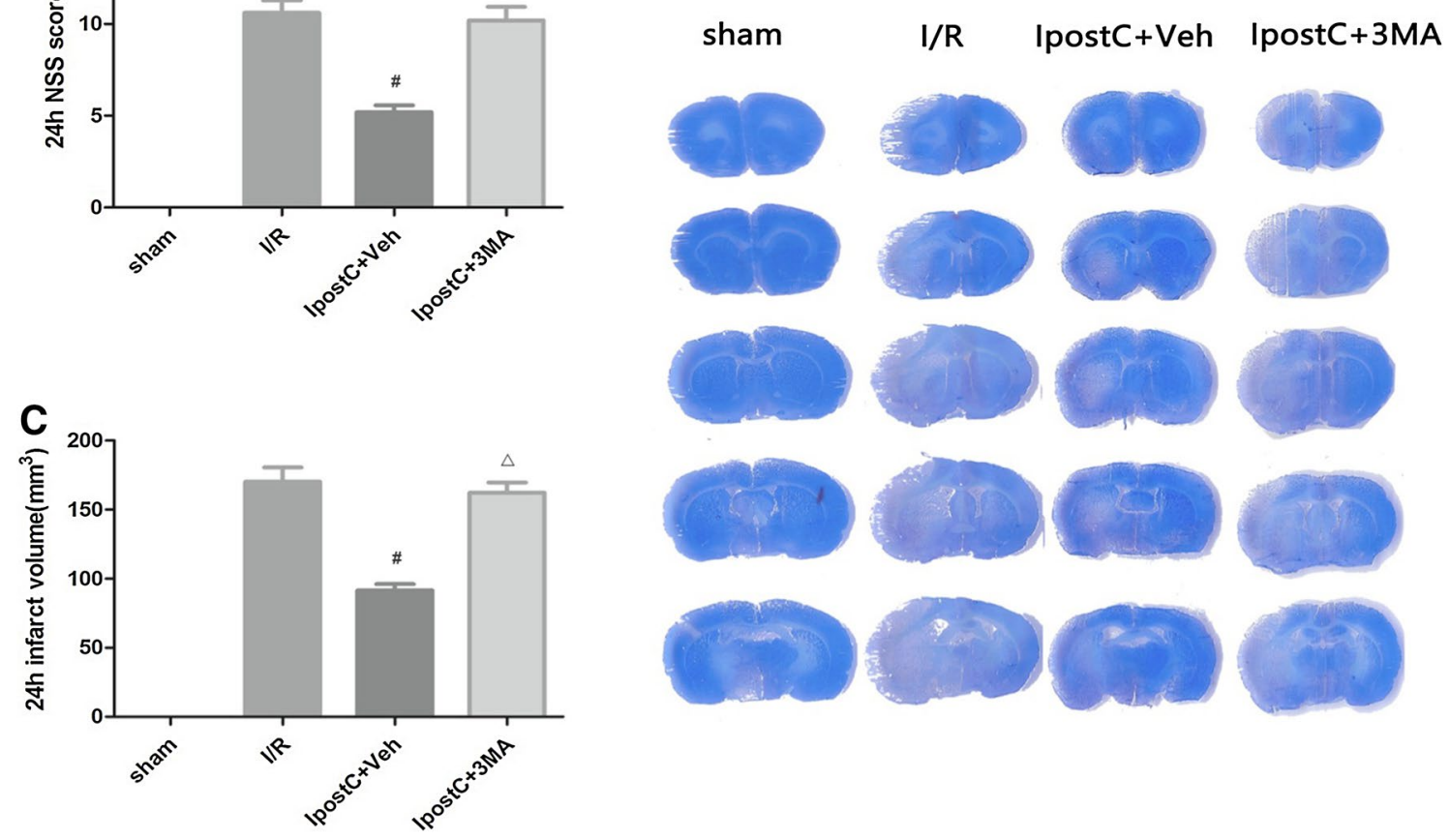

Fig. 2 An inhibitor of autophagy attenuated the protective effect of IpostC against cerebral I/R injury. a Neurologic function assessed using the modified Neurologic Severity Score (mNSS) in the sham, $\mathrm{I} / \mathrm{R}$, IpostC+Veh and IpostC+3-methyladenine (3MA) groups. Data presented as the mean \pm SD $(n=9$ each group). b Representa-

followed by the Tukey's post-hoc test. $P<0.05$ was considered statistically significant.

\section{Results}

\section{Effects of IpostC on Time-Dependent Expression Levels of LC3, p62 and Beclin-1 in the Cerebral Cortex Following Cerebral I/R Injury}

To determine the extent of autophagy activation in the MCAO model of focal cerebral ischemia, the cortical expressions of three autophagic markers, LC3, p62 and beclin-1, were determined at $1,6,12,24$, or $48 \mathrm{~h}$ postischemia (Fig. 1). Western blot revealed that the LC3-II/ LC3-I ratio in the I/R group started to raise by $6 \mathrm{~h}$, reaching significantly high levels at $12 \mathrm{~h}(P<0.05$ vs. sham group), peaked at $24 \mathrm{~h}(P<0.05$ vs. sham group) and was maintained at a high level at $48 \mathrm{~h}$ post-ischemia $(P<0.05$ vs. sham group; $P>0.05$ vs. I/R group at 24 h, Fig. 1a). The expression of p62 in the I/R group also increased significantly to peak at $6 \mathrm{~h}(P<0.01$ vs. sham group). The p62 levels subsequently decreased but remained higher than that in the sham group at 12 and $24 \mathrm{~h}$ (both $P<0.05$ tive images of brain slices stained with cresyl violet in different groups. Regions of cerebral infarction are stained white. c Infarct volume quantified using ImageJ software. Data presented as the mean \pm standard deviation (SD) $\left(\mathrm{n}=6\right.$ each group). ${ }^{\#} P<0.05$ vs. I/R group; ${ }^{\triangle} P<0.05$ vs. IpostC + Veh group

vs. sham group), before returning to baseline levels at $48 \mathrm{~h}$ (Fig. 1c). Furthermore, the expression of beclin-1 in the I/R group peaked at $6 \mathrm{~h}(P<0.05$ vs. sham group $)$. The beclin-1 levels subsequently returned to baseline levels by 24 h (both $P>0.05$ vs. sham group) (Fig. 1e).

In the IpostC group, the LC3-II/LC3-I ratio started to increase at $6 \mathrm{~h}(P<0.05$ vs. sham group), peaked at $12 \mathrm{~h}$ $(P<0.05$ vs. sham group), but was not fully maintained at $48 \mathrm{~h}$ post-ischemia $(P<0.05$ vs. IpostC $24 \mathrm{~h})($ Fig. $1 \mathrm{~b})$. As shown in Fig. 1d, p62 expression in the IpostC group also peaked at $6 \mathrm{~h}(P<0.01$ vs. sham group), but the expression of p62 subsequently decreased progressively to reach a level at $48 \mathrm{~h}$ that was significantly lower than that in the sham group $(P<0.001 ;$ Fig. $1 \mathrm{~d})$. The fall in the p62 levels in the IpostC group is consistent with increased autophagic degradation of p62 resulting from an enhancement of autophagy by IpostC. In addition, the expression of beclin-1 in the IpostC group peaked at $1 \mathrm{~h}$ and remained high at 6 and $12 \mathrm{~h}(P<0.05$ vs. sham group). The beclin-1 levels subsequently returned to baseline levels at $24 \mathrm{~h}$ ( $P>0.05$ vs. sham group) and reached lower values than in the control group at $48 \mathrm{~h}(P<0.05$ vs. sham group $)$ (Fig. 1f). 
A
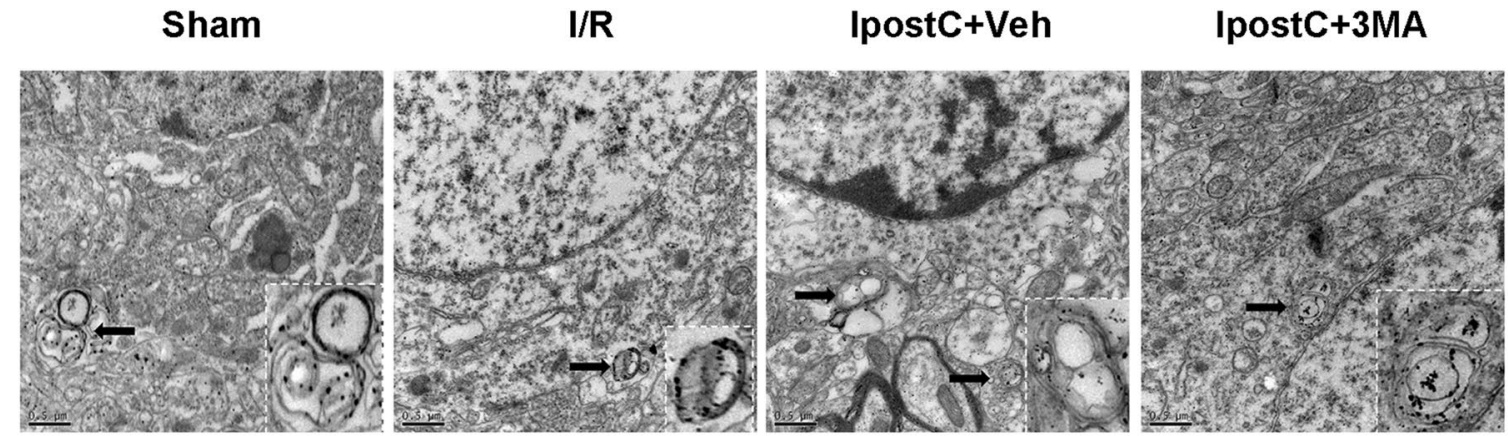

ALs

B

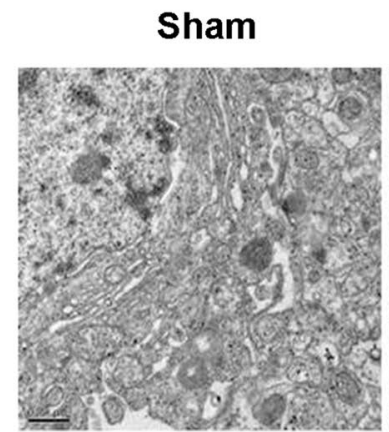

C

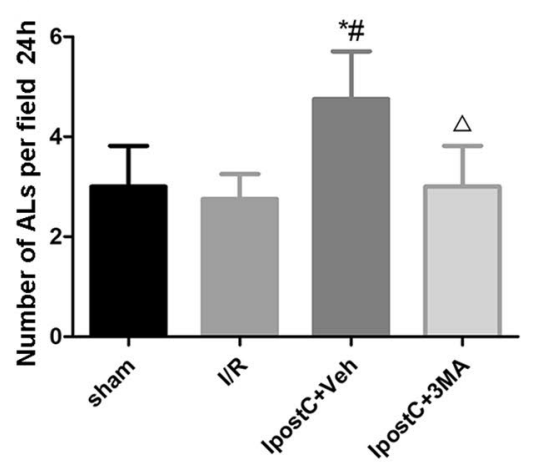

D
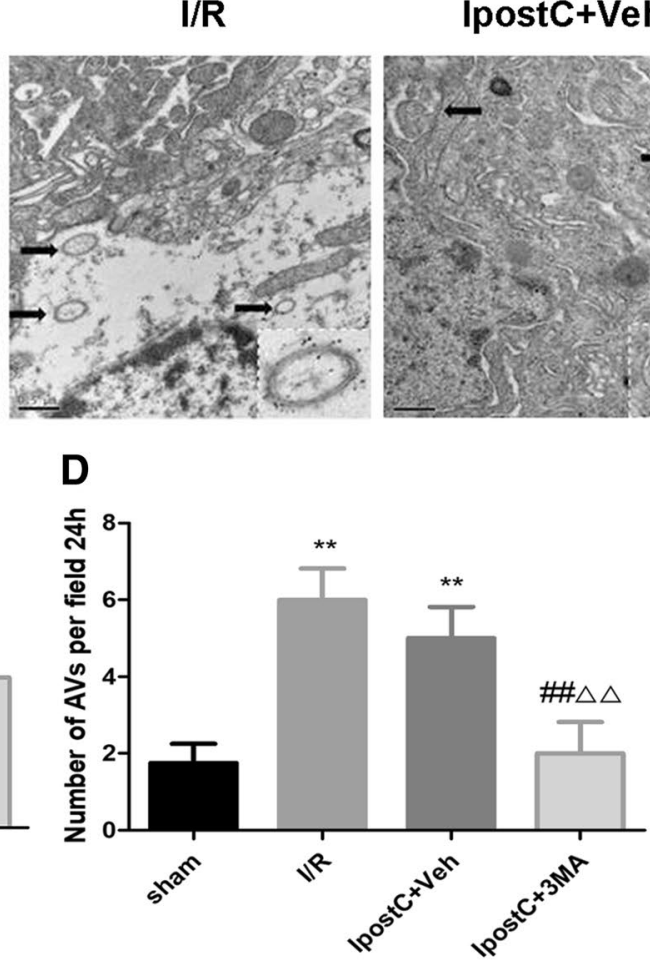

IpostC+Veh

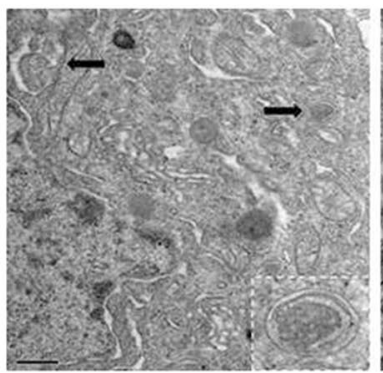

IpostC+3MA

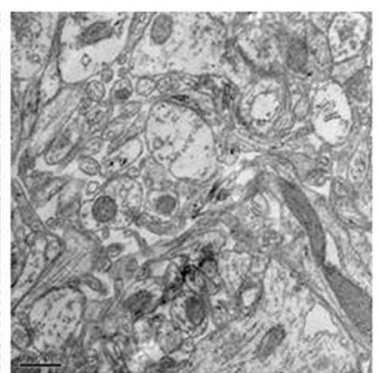

E

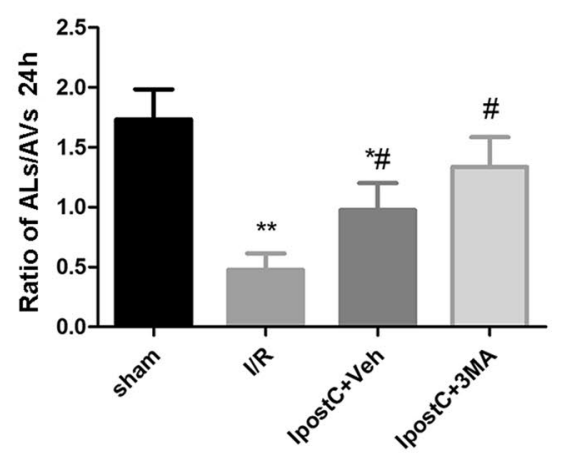

Fig. 3 Effects of IpostC on formation of autophagosomes and autolysosomes in the cerebral cortex following I/R injury. a Representative transmission electron microscopy images of autolysosomes (ALs) in the various groups. Black arrows indicate ALs. b Representative transmission electron microscopy images of autophagosomes (AVs) in the various groups. Black arrows indicate AVs. Scale bars: $0.5 \mu \mathrm{m}$. Insets in the pictures show enlarged autolysosomes or

\section{An Inhibitor of Autophagy Attenuated the Protective Effect of IpostC Against Cerebral I/R injury}

mNSS score after $24 \mathrm{~h}$ of reperfusion was significantly lower in the IpostC+Veh group than in the I/R group or IpostC+3MA group (both $P<0.05$, Fig. 2a). Furthermore, staining of brain sections with cresyl violet revealed areas of cerebral infarction (stained white) in the I/R, IpostC $+3 \mathrm{MA}$, and IpostC+Veh groups but not in the sham group (Fig. 2b). Quantification of the staining showed that the infarct volume autophagosomes which were indicated by black arrows. c Quantitative analysis of the number of ALs per field in the various groups. d Quantitative analysis of the number of AVs per field in the various groups. e Quantitative analysis of the AL/AV ratio in the various groups. Data presented as the mean \pm SEM ( $n=3$ each group). $* P<0.05, * * P<0.01$ vs. sham group; ${ }^{\#} P<0.05,{ }^{\# \#} P<0.01$ vs. I/R group; ${ }^{\triangle} P<0.05,{ }^{\triangle} P<0.01$ vs. IpostC + Veh group

in the IpostC+Veh group was significantly lower than that in the I/R group or IpostC+3MA group (both $P<0.05$, Fig. $2 \mathrm{c}$ ).

\section{Effects of IpostC on Formation of Autophagosomes and Autolysosomes in the Cerebral Cortex Following I/R Injury}

TEM was used to observe autophagosomes and autolysosomes in order to monitor the induction of autophagy (Fig. 3). Compared with the sham and IpostC+3MA groups, the number of autolysosomes per field was significantly 

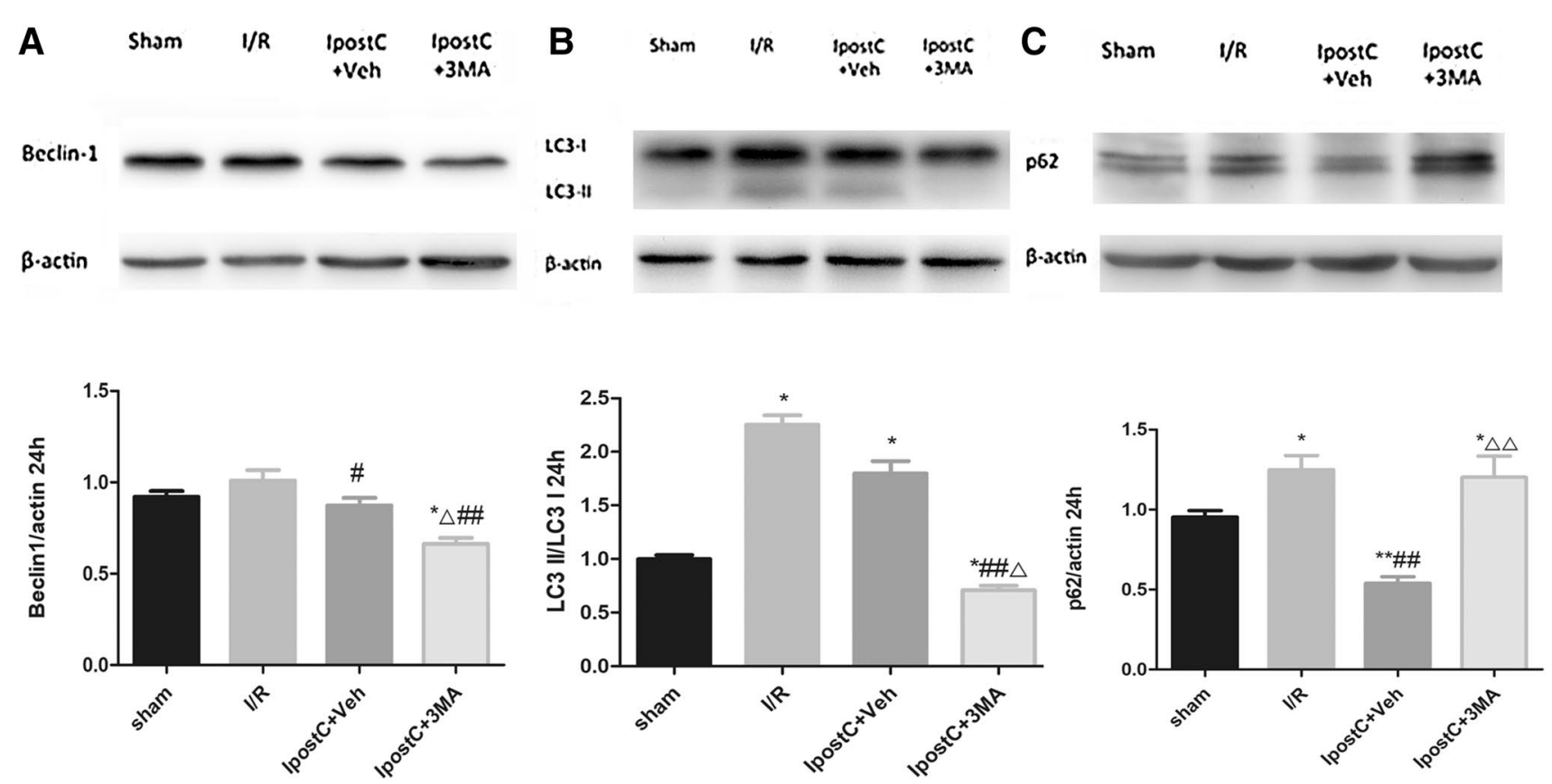

D SHAM I/R

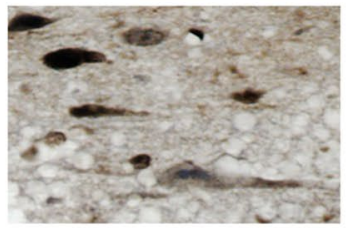

IpostC+Veh

IpostC+3MA

Beclin1
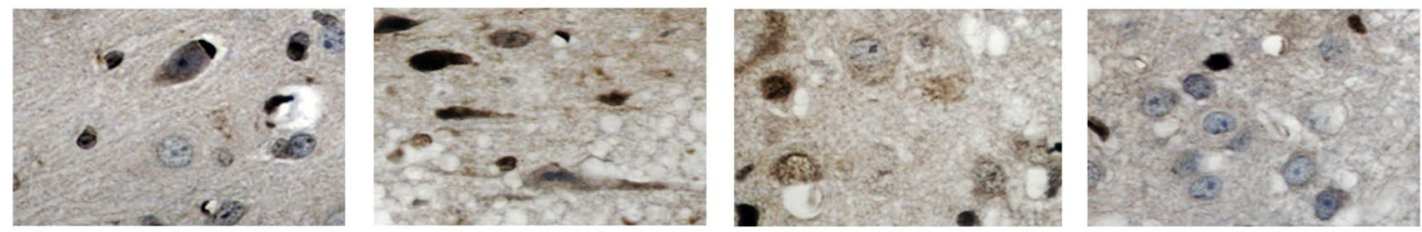

LC3
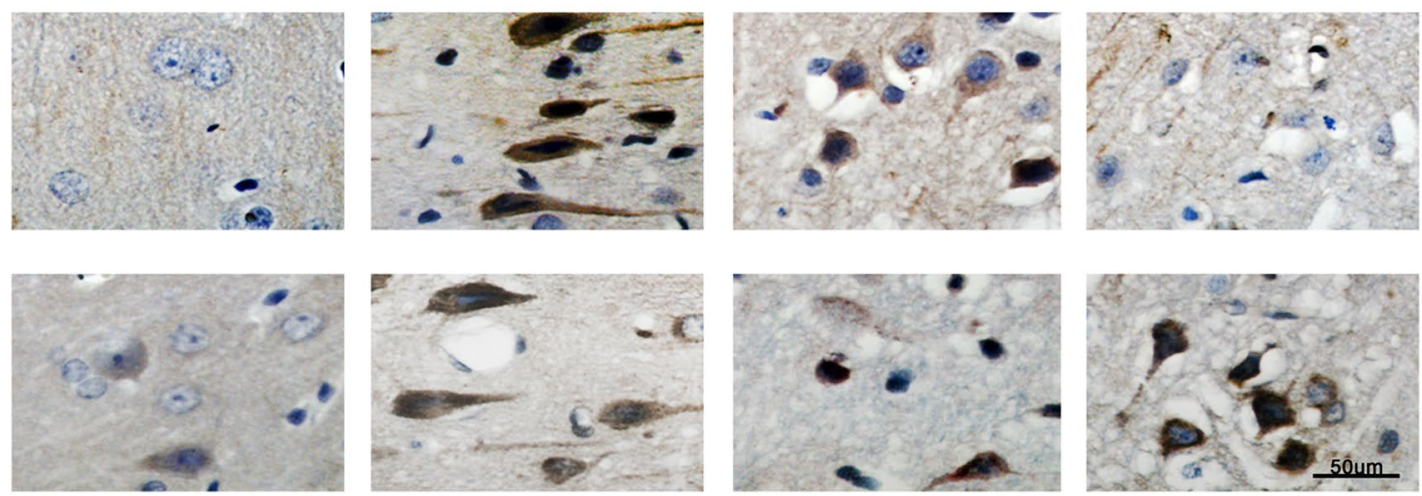

Fig. 4 Effects of IpostC on expression levels of autophagy-related proteins (beclin-1, LC3, p62) in the cerebral cortex following I/R injury. Western blot was used to determine the beclin-1 (a), LC3-II/ LC3-I ratio (b) and p62 (c) protein expression levels in the various groups. d Representative immunohistochemical images showing

higher in the IpostC+Veh group $(P<0.05$ vs. sham group, $\mathrm{I} / \mathrm{R}$ group, and IpostC+3MA group) (Fig. 3c). Compared with the sham and the IpostC+3MA groups, the number of autophagosomes per field was significantly higher in both the $\mathrm{I} / \mathrm{R}$ and IpostC+Veh groups (both $P<0.01$ ) (Fig. 3d). Similarly, the ratio of autolysosomes to autophagosomes was significantly lower in the $\mathrm{I} / \mathrm{R}$ group and IpostC+Veh the localization of beclin-1, LC3, and p62 in the cerebral cortex of the hemisphere ipsilateral to the ischemic region. Scale bar: $50 \mu \mathrm{m}$. Data presented as the mean \pm SEM ( $n=3$ each group). $* P<0.05$, ${ }^{* *} P<0.01$ vs. sham group; ${ }^{\#} P<0.05$, ${ }^{\# \#} P<0.01$ vs. I/R group; ${ }^{\triangle} P<0.05,{ }^{\triangle} \triangle P<0.01$ vs. Ipost $C+$ Veh group

group than in the sham and the IpostC+3MA groups (all $P<0.05)$. The autolysosome to autophagosome ratio was slightly higher in the IpostC+Veh group than in the I/R group $(P<0.05$; Fig. 3e). Notably, the ratio of autolysosomes to autophagosomes in the IpostC $+3 \mathrm{MA}$ group was significantly higher than that in the $\mathrm{I} / \mathrm{R}$ group $(P<0.05)$ (Fig. 3e). 
A

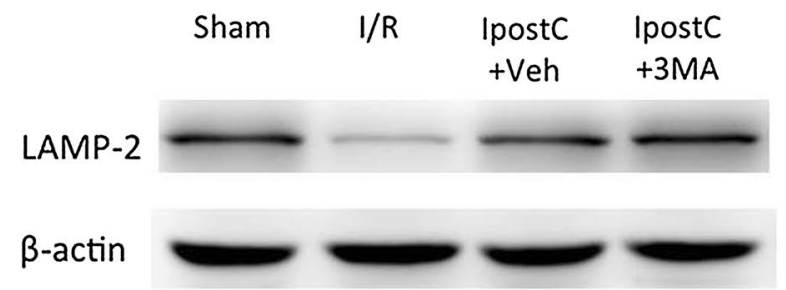

B

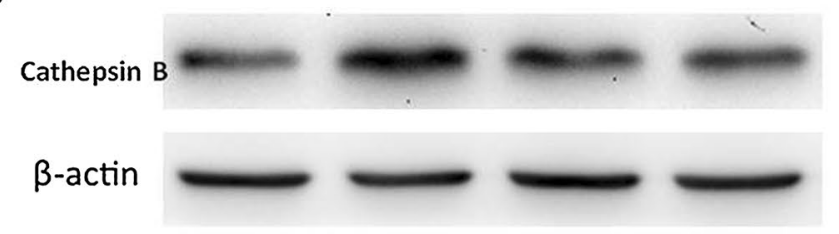

C

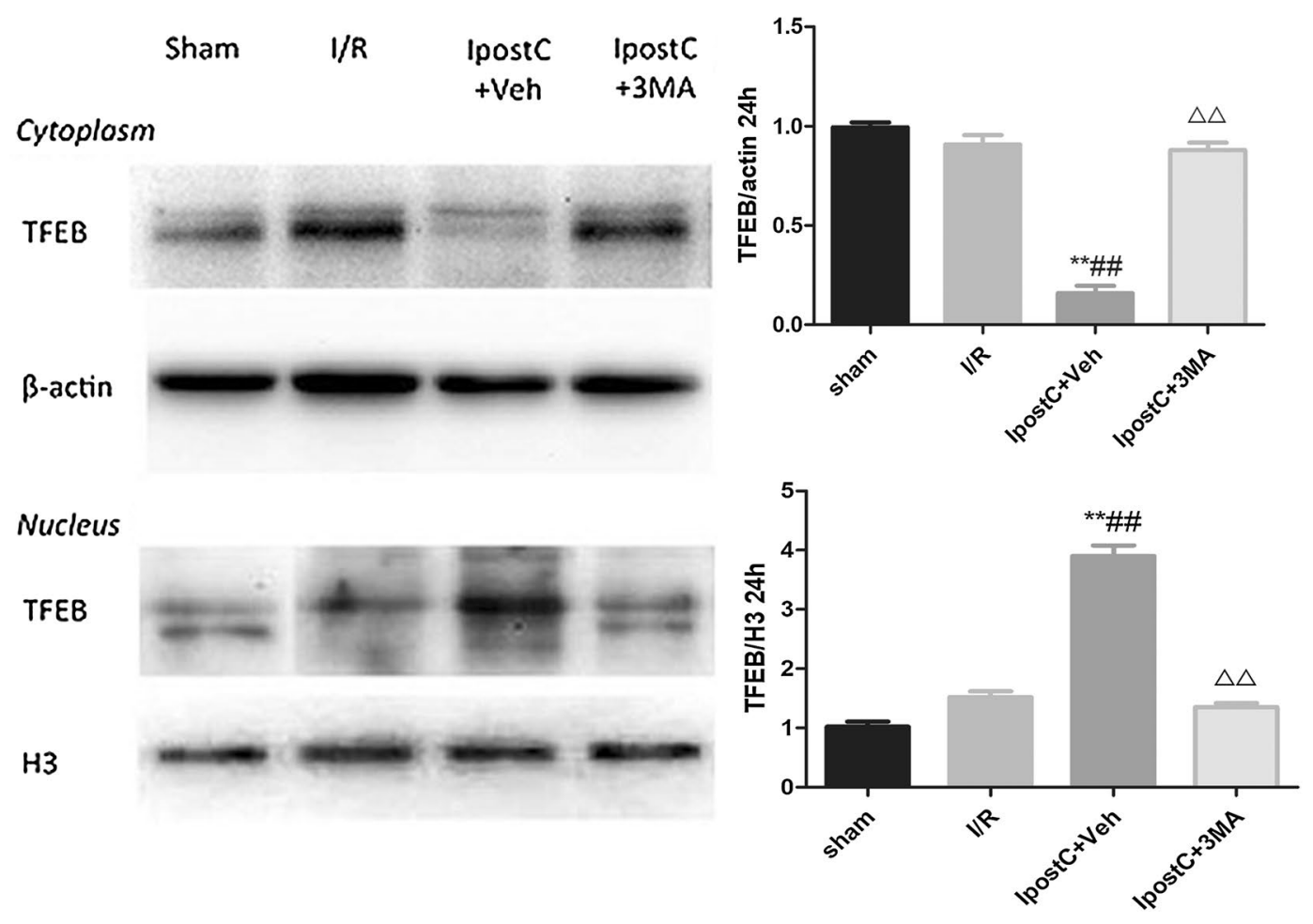

Fig. 5 Effects of IpostC on expression levels of autophagy-related proteins (LAMP-2, Cathepsin B and TFEB) in the cerebral cortex following I/R injury. LAMP-2 (a) and Cathepsin B (b) protein expression levels in the various groups were determined by western blot. c Subcellular fractionation of the cerebral cortex was employed
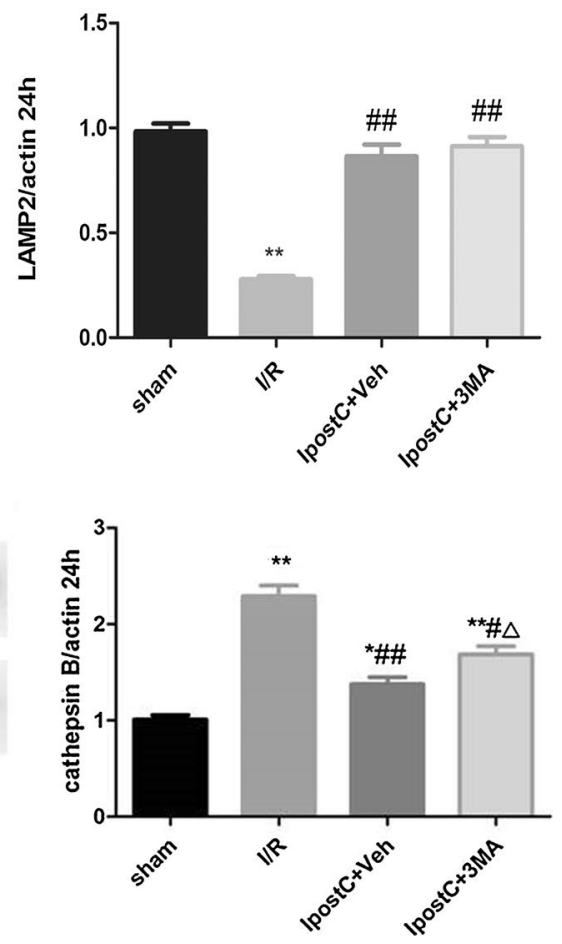

to determine the subcellular location of TFEB by Western blot. Data presented as the mean \pm SEM ( $\mathrm{n}=3$ each group). ${ }^{*} P<0.05$, ${ }^{* *} P<0.01$ vs. sham group; ${ }^{\#} P<0.05,{ }^{\# \#} P<0.01$ vs. I/R group; ${ }^{\triangle} P<0.05,{ }^{\triangle} \triangle P<0.01$ vs. IpostC + Veh group 

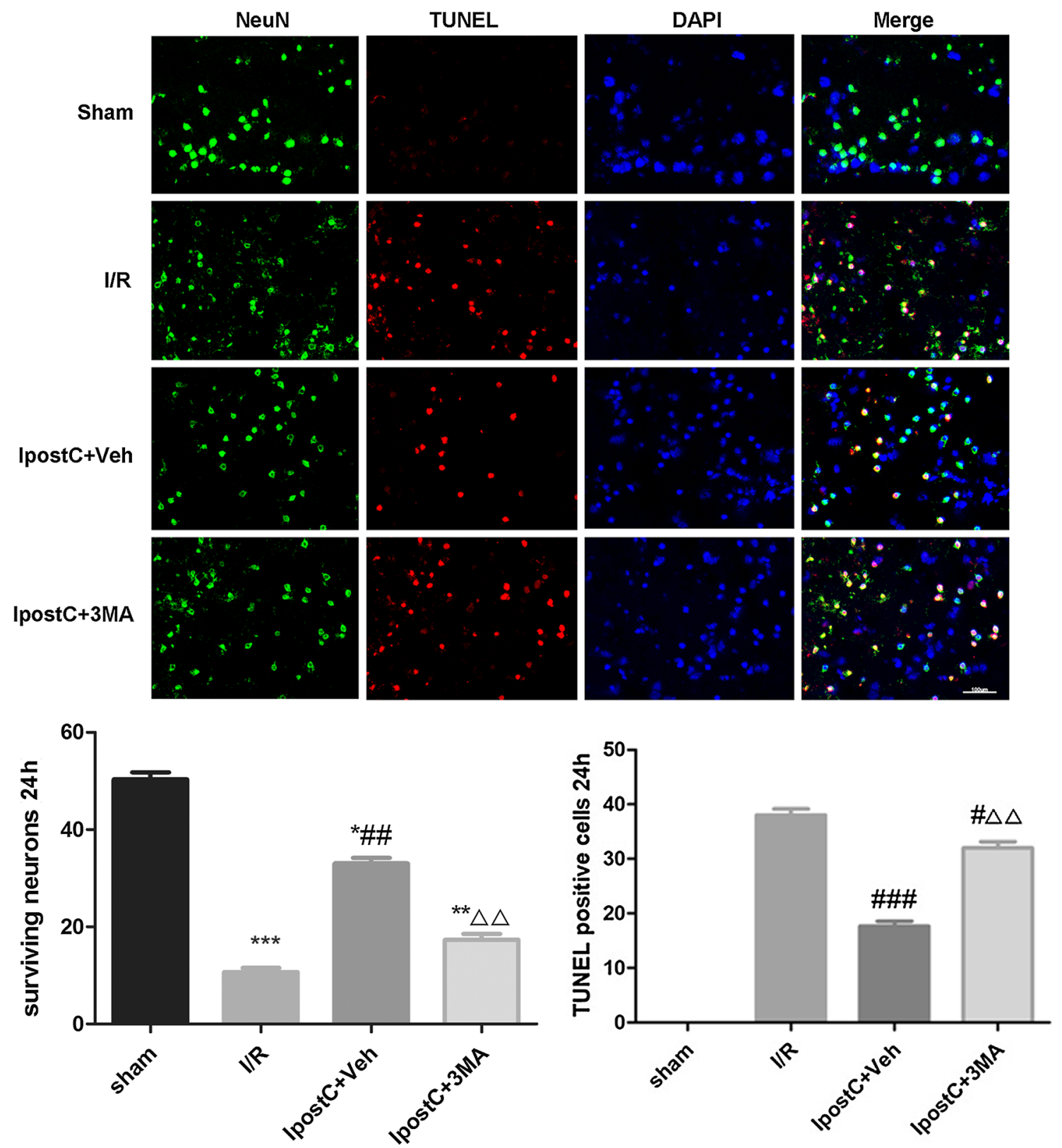

Fig. 6 Effect of IpostC on I/R-induced apoptosis in the cerebral cortex. Representative images showing immunofluorescent staining for the neuronal marker NeuN (green) and the TUNEL assay (red) in sections of the cerebral cortex. DAPI (blue): nucleus. Magnification:

\section{Effects of IpostC on Expression Levels of Autophagy-Related Proteins in the Cerebral Cortex Following I/R Injury}

Western blotting (Fig. 4) revealed that cerebral I/R upregulated the levels of autophagy-related proteins (LC3, beclin-1, and p62) in the cerebral cortex. IpostC partially reversed the increased levels of beclin-1 $(P<0.05)$ and p62 $(P<0.01)$ at $24 \mathrm{~h}$ after I/R (Fig. 4a, c), but not LC3-II/LC3-I $(P>0.05)$ (Fig. 4b). Furthermore, the expressions of beclin-1 and $\times 40$. Scale bar: $100 \mu \mathrm{m}$. Data presented as the mean $\pm \operatorname{SEM}(n=3$ each group). $* P<0.05, * * P<0.01, * * * P<0.001$ vs. sham group; ${ }^{\#} P<0.05$, ${ }^{\# \#} P<0.01$, ${ }^{\# \# \#} P<0.001$ vs. I/R group; ${ }^{\triangle} P<0.01$ vs. IpostC+Veh group

LC3 were lower in the IpostC $+3 \mathrm{MA}$ group than in the IpostC+Veh group (both $P<0.05$ ) (Fig. $4 \mathrm{a}$, b), whereas that of $\mathrm{p} 62$ (which is degraded by autophagosomes) was higher $(P<0.01)$ (Fig. $4 \mathrm{c})$, consistent with the inhibition of autophagy by $3 \mathrm{MA}$. These results were supported by the immunohistochemistry experiment (Fig. 4d).

The protein expression levels of LAMP-2, cathepsin $\mathrm{B}$, and TFEB were further determined by western blot. The LAMP-2 protein levels were significantly lower in the $\mathrm{I} / \mathrm{R}$ group than in the sham group at $24 \mathrm{~h}$ post-ischemia 

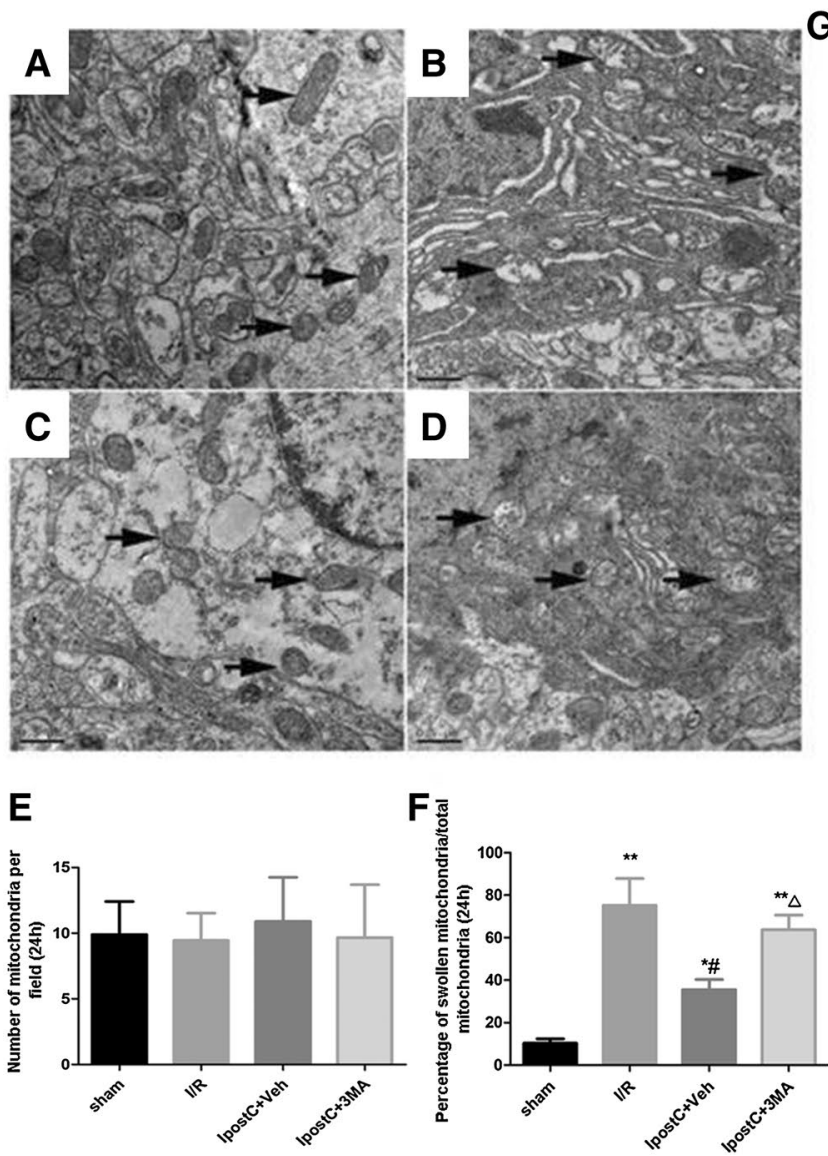

Fig. 7 Effect of IpostC on mitochondrial number and morphology and mitochondria-dependent apoptosis related protein (Bax and cytochrome-c) expression levels in the cerebral cortex following $\mathrm{I} / \mathrm{R}$ injury. (a-d) Representative transmission electron microscopy images to show mitochondrial morphology in the sham (a), I/R (b), IpostC+Veh (c) and IpostC+3MA (d) groups. Mitochondrial vacuoles are indicated by arrows. Scale bars: $0.5 \mu \mathrm{m}$. e Quantitative analysis of the number of mitochondria per field. f Quantitative analysis of

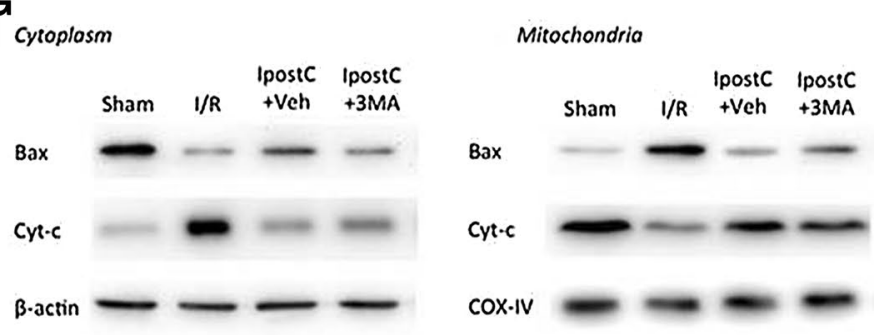

H Cytoplosm Mitochondria
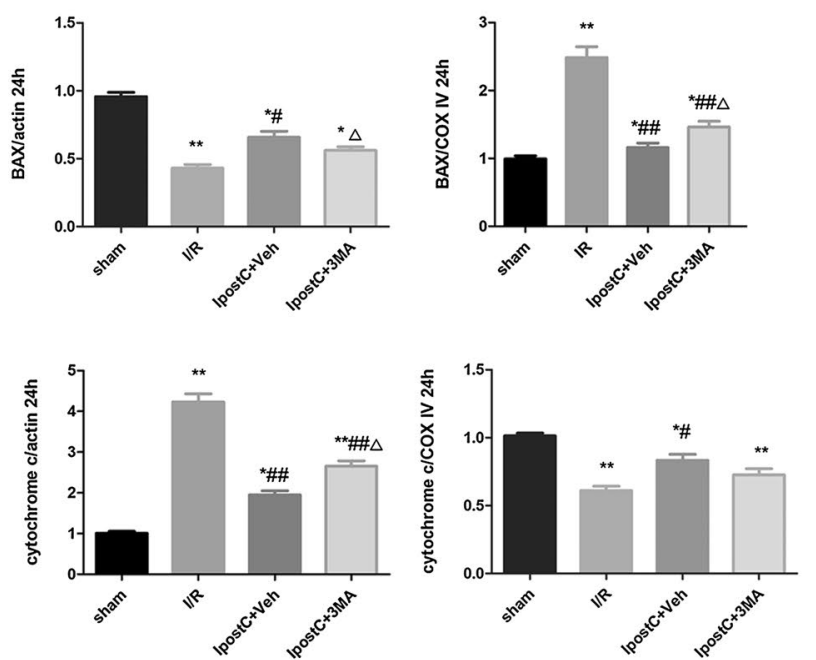

the ratio of swollen mitochondria to total mitochondria. $\mathbf{g}$ Representative immunoblots showing the distribution of Bax and cytochrome-c in the cytoplasm and mitochondria in the various groups, determined after subcellular fractionation had been performed. h Quantitative analysis of the cytoplasmic and mitochondrial levels of Bax and cytochrome-c. Data presented as the mean \pm SEM ( $n=3$ each group). ${ }^{*} P<0.05,{ }^{*} P<0.01$ vs. sham group; ${ }^{\#} P<0.05,{ }^{\# \#} P<0.01$ vs. I/R group; ${ }^{\triangle} P<0.05$ vs. IpostC + Veh group

\section{Effect of IpostC on I/R-Induced Apoptosis in the Cerebral Cortex}

LAMP-2 was not observed in the IpostC+Veh and the IpostC+3MA groups $(P>0.05)$ (Fig. 5a), indicating that IpostC attenuated I/R-induced LAMP-2 downregulation by enhancing autophagy. The cathepsin B protein levels were significantly higher in the $\mathrm{I} / \mathrm{R}$ group than in the sham group at $24 \mathrm{~h}$ post-ischemia $(P<0.01 ; \mathrm{Fig}$. $5 \mathrm{~b})$. The I/R-induced upregulation of cathepsin $B$ was observed in the IpostC + Veh and the IpostC $+3 \mathrm{MA}$ groups $(P<0.05$ vs. sham group), but the levels were lower than in the I/R group $(P<0.05)$ (Fig. 5b). Subcellular fractionation experiments were performed to address whether IpostC and autophagy were linked to nuclear translocation of TFEB. There was a significant increase in nuclear TFEB after IpostC treatment that was blocked by the administration of 3MA $(P<0.01$; Fig. $5 \mathrm{c})$.
TUNEL assay showed that I/R induced the apoptosis of neurons in the cerebral cortex (Fig. 6). IpostC attenuated I/R-induced neuronal apoptosis $(P<0.001)$ and $3 \mathrm{MA}$ inhibited this effect of IpostC $(P<0.01)$ (Fig. 6).

Mitochondrial number and morphology were also assessed using TEM (Fig. 7a-d). The total number of mitochondria did not differ significantly among the four groups (Fig. 7e), but the ratio of swollen mitochondria to total mitochondria was significantly higher in the $\mathrm{I} / \mathrm{R}$ $(P<0.01)$, IpostC + Veh $(P<0.05)$, and IpostC $+3 \mathrm{MA}$ $(P<0.01)$ groups than in the sham group (Fig. 7f). The ratio of swollen mitochondria to total mitochondria in the IpostC + Veh group was significantly lower than that 
in both the I/R and IpostC $+3 \mathrm{MA}$ groups (both $P<0.05$; Fig. 7f).

To explore whether IpostC and autophagy were associated with mitochondrial translocation of Bax, subcellular fractionation experiments were performed (Fig. 7g). The mitochondrial Bax levels were increased significantly after $\mathrm{I} / \mathrm{R}(P<0.01)$, and IpostC attenuated mitochondrial translocation of Bax, whereas 3MA inhibited this effect of IpostC $(P<0.05$; Fig. 7h).

Mitochondrial translocation of cytochrome-c was also shown by subcellular fractionation experiments (Fig. $7 \mathrm{~g}$ ). The cytoplasmic cytochrome-c levels were increased in the I/R group compared with the sham group $(P<0.01 ;$ Fig. 7 h $)$, indicating mitochondrial dysfunction. Mitochondrial release of cytochrome-c after $\mathrm{I} / \mathrm{R}$ was reduced by IpostC $(P<0.01$; Fig. 7h), while 3MA attenuated this effect of IpostC $(P<0.05$; Fig. 7h).

\section{Discussion}

Stroke is associated with high morbidity and mortality, highlighting the need for novel clinical approaches to treating this disease. IpostC applied at the onset of reperfusion has been reported to ameliorate I/R injury [44]. Multiple mechanisms have been suggested to contribute to the neuroprotective mechanisms of IpostC, including regulation of synaptic signaling, attenuation of oxidative stress and inflammation, maintenance of mitochondrial integrity, inhibition of endoplasmic reticulum stress, activation of the phosphoinositide 3-kinase/Akt pathway, inhibition of apoptosis and protection of the neurovascular unit [21-25, 27, 28]. Nevertheless, it is unclear whether autophagy during early reperfusion is influenced by IpostC or plays a role in the effects of IpostC. The results of the present study strongly suggest that IpostC improved neurologic function and reduced infarct volume after I/R injury. These effects of IpostC were inhibited by $3 \mathrm{MA}$, an inhibitor of autophagy. Indeed, autophagosome formation was increased in the I/R and IpostC+Veh groups, but not in the IpostC+3MA group. These results support the hypothesis stating that autophagy participates in the protective effect of IpostC on I/R injury, as supported by previous studies performed in different models and organs [21-25, $27,28]$.

Compared with the sham group, the I/R group showed an increased number of autophagosomes, higher levels of $\mathrm{p} 62$, and lower levels of LAMP-2. These findings are consistent with dysfunctional autophagic flux and clearance during the reperfusion period, possibly due to impaired fusion of autophagosomes with lysosomes or impaired autolysosomal degradation. IpostC significantly reduced infarct volume and improved neurologic function, and these effects were attenuated by 3MA (an inhibitor of autophagy), suggesting that activation of autophagy was involved in the beneficial effects of IpostC. Furthermore, the low levels of the p62 protein, high levels of the LAMP-2 protein, and nuclear translocation of TFEB in the IpostC group suggested that IpostC promoted autophagic flux that progressed to its completion. In addition, cathepsin B levels were higher in the $\mathrm{I} / \mathrm{R}$ group, while the increase was attenuated by IpostC. This is supported by previous studies showed that cathepsin $\mathrm{B}$ is involved in autophagy [15], and that inhibition of cathepsin B contributed to neuroprotection against cerebral ischemic injury [14]. The detection of apoptosis-related proteins revealed that IpostC prevented the mitochondrial translocation of Bax and reduced cytochrome-c release from mitochondria associated with I/R. Moreover, 3MA inhibited these effects of IpostC. Taken together, these results strongly suggest that the autophagic pathway following I/R injury may be dysfunctional and that IpostC ameliorates cerebral I/R injury through activation of autophagy.

Autophagic flux encompasses the entire process of autophagy, including the formation of autophagosomes, the transport of substrates to lysosomes, the degradation of substrates, and the release of macromolecules to the cytoplasm [45]. Therefore, it is necessary to monitor autophagic flux in order to detect autophagic activity [45]. Membrane-bound LC3-II can be used as a marker of autophagy because it is retained in mature autophagosomes and is not released until fusion with the lysosome [46]. p62 participates in the selective degradation of ubiquitinated proteins by autophagy [47], thus the expression of p62 directly reflects the level of autophagic clearance. The levels of both LC3-II and p62 proteins need to be measured in order to adequately monitor the autophagic flux [48].

Previous research indicated that ischemic stroke might lead to the activation of autophagy through mechanisms that include a depletion of nutrients, oxidative stress, and protein misfolding [49]. The activation of autophagy after ischemia has been detected in models of focal cerebral ischemia [50] and global cerebral ischemia [51]. Wei et al. [28] observed that IpostC activated autophagy and inhibited myocardial cell apoptosis in rat hearts subjected to I/R injury. Hao et al. [24] reported that $3 \mathrm{MA}$ suppressed the cardioprotective effects of IpostC against I/R injury and suggested that IpostC promoted autophagy partially via activation of the neuronal nitric oxide synthase/adenosine monophosphate-activated protein kinase (AMPK)/mechanistic target of rapamycin (mTOR) pathway. Consistent with the aforementioned study, Guo et al. [23] highlighted the crucial role of autophagy in the protective mechanisms of IpostC in cardiomyocytes, through the regulation of beclin- 1 and AMPK/mTOR signaling pathways. Using the transient MCAO model of cerebral I/R, Qi et al. [25, 26] found that remote limb IpostC activated autophagy and reduced cell death through the AKT/ GSK3 $\beta$ pathway and Bcl-2 phosphorylation, and the authors 
suggested that this may contribute to the attenuation of mitochondrial damage by IpostC. Zhang et al. [31] reported that inhibition of autophagy during the reperfusion phase worsened neuronal death in an in vivo model (MCAO in mice) and an in vitro model (cultured cortical neurons) of I/R.

The protective role of autophagy was attributed to mitophagy-related mitochondrial clearance (possibly involving the E3 ligase, PARK2) and inhibition of downstream apoptosis. On the other hand, a published study yielded contradictory results. Indeed, Gao et al. [22] created a focal cerebral ischemia model involving permanent distal MCAO plus transient bilateral CCA occlusion and reported that IpostC inhibited the upregulation of LC3/beclin-1 and reversed the reduction of p62 caused by ischemia. Furthermore, rapamycin (an inducer of autophagy) attenuated the effects of IpostC while 3MA induced neuroprotection [22]. We suggest that two important factors might contribute to the discrepancies between the latter study and ours. First, we performed IpostC using a different experimental method. Second, the markers detected in this study were different from those used in the study of Gao et al. [22], in order to more clearly delineate changes in the autophagy pathway. After formation, autophagosomes fuse with lysosomes for subsequent clearance [22]. Impaired clearance of autophagosomes could result in their accumulation, which could be incorrectly interpreted as enhanced autophagy. Therefore, we detected lysosomal activity by measuring the expression of LAMP-2, a major protein on the lysosomal membrane that plays an important role in the fusion of autophagosomes and lysosomes [18]. In addition, we measured the nuclear translocation of TFEB protein, which regulates lysosomal biogenesis and autophagy. TFEB promotes the autophagosomal-lysosomal fusion for protein clearance and prevents accumulation of autophagosomes [52, 53]. TFEB can upregulate the expression of nearly two-thirds of autophagy-lysosome genes and its overexpression shows potential therapeutic effects in cardiovascular disease by rescuing lipid-induced lysosomal dysfunction [16, 52, 54] as well as enhancing lipolysis [55]. Normally, TFEB is located in the cytosol and on the lysosomal surface, where it interacts with mTOR in its inactive phosphorylated form; however, in response to stimuli, TFEB translocates to the nucleus [56].

Our observations indicate that IpostC can stimulate functional autophagy that protects against cerebral I/R injury, and that the underlying mechanism may involve translocation of TFEB to the nucleus and transcription of target genes that promote autophagic flux. The clearance of impaired mitochondria by autophagy is beneficial for neuronal survival, as previously shown [25, 34]. The ratio of swollen mitochondria to total mitochondria in the IpostC + Veh group was significantly lower than that in both $\mathrm{I} / \mathrm{R}$ and IpostC+3MA groups. The results of cytochrome-c and
BAX were concordant with enhanced autophagy of mitochondria. Qi et al. [25] showed that Bcl-2 phosphorylation and disruption of the Bcl-2/beclin-1 complex was essential to autophagy triggering and reduced mitochondrial damage after cerebral ischemia. Baek et al. [34] showed that carnosine used as a protective agent after cerebral ischemia played its beneficial roles in part through mitochondrial protection and autophagy attenuation. Nevertheless, additional study is still necessary to examine and understand the mechanisms involved in the protective effects of IpostC against I/R injury.

In summary, the present study strongly suggests that the neuroprotection induced by IpostC against I/R injury is mediated, at least in part, by promotion of autophagy during early reperfusion. The autophagy pathway may be a novel target for the development of new clinical treatments for ischemic stroke.

Funding This work was supported by the National Natural Science Foundation of China (31371065) and Natural Science Foundation of Shanghai (No. 17ZR1425800). This work was supported by Medical Professional Cross Research Fund Project of Shanghai Jiao Tong University (YG2015MS14).

\section{Compliance with Ethical Standards}

Conflict of interest The authors declare that they have no conflict of interest.

Research involving Animals All experimental procedures were approved by the Institutional Animal Care and Use Committee of Shanghai Jiao Tong University, Shanghai, China.

Informed consent Not applicable.

Open Access This article is distributed under the terms of the Creative Commons Attribution 4.0 International License (http://creativeco mmons.org/licenses/by/4.0/), which permits unrestricted use, distribution, and reproduction in any medium, provided you give appropriate credit to the original author(s) and the source, provide a link to the Creative Commons license, and indicate if changes were made.

\section{References}

1. Writing Group M, Mozaffarian D, Benjamin EJ, Go AS, Arnett DK, Blaha MJ, Cushman M, Das SR, de Ferranti S, Despres JP, Fullerton HJ, Howard VJ, Huffman MD, Isasi CR, Jimenez MC, Judd SE, Kissela BM, Lichtman JH, Lisabeth LD, Liu S, Mackey RH, Magid DJ, McGuire DK, Mohler ER 3rd, Moy CS, Muntner P, Mussolino ME, Nasir K, Neumar RW, Nichol G, Palaniappan L, Pandey DK, Reeves MJ, Rodriguez CJ, Rosamond W, Sorlie PD, Stein J, Towfighi A, Turan TN, Virani SS, Woo D, Yeh RW, Turner MB, American Heart Association Statistics C, Stroke Statistics S (2016) Heart disease and stroke statistics-2016 update: a report from the American Heart Association. Circulation 133(4):e38-e360. https://doi.org/10.1161/CIR.000000000000035 0 
2. Wang W, Jiang B, Sun H, Ru X, Sun D, Wang L, Wang L, Jiang Y, Li Y, Wang Y, Chen Z, Wu S, Zhang Y, Wang D, Wang Y, Feigin VL, Investigators NE-C (2017) Prevalence, incidence, and mortality of stroke in China: results from a nationwide population-based survey of 480687 adults. Circulation 135(8):759-771. https://doi. org/10.1161/CIRCULATIONAHA.116.025250

3. Steiger HJ, Hanggi D (2007) Ischaemic preconditioning of the brain, mechanisms and applications. Acta Neurochir (Wien) 149(1):1-10. https://doi.org/10.1007/s00701-006-1057-1

4. Zhao H, Sapolsky RM, Steinberg GK (2006) Interrupting reperfusion as a stroke therapy: ischemic postconditioning reduces infarct size after focal ischemia in rats. J Cereb Blood Flow Metab 26(9):1114-1121. https://doi.org/10.1038/sj.jcbfm.9600348

5. Gao X, Ren C, Zhao H (2008) Protective effects of ischemic postconditioning compared with gradual reperfusion or preconditioning. J Neurosci Res 86(11):2505-2511. https://doi.org/10.1002/ jnr.21703

6. Levine B, Kroemer G (2008) Autophagy in the pathogenesis of disease. Cell 132(1):27-42. https://doi.org/10.1016/j. cell.2007.12.018

7. Pattingre S, Tassa A, Qu X, Garuti R, Liang XH, Mizushima N, Packer M, Schneider MD, Levine B (2005) Bcl-2 antiapoptotic proteins inhibit Beclin 1-dependent autophagy. Cell 122(6):927939. https://doi.org/10.1016/j.cell.2005.07.002

8. Kabeya Y, Mizushima N, Ueno T, Yamamoto A, Kirisako T, Noda T, Kominami E, Ohsumi Y, Yoshimori T (2000) LC3, a mammalian homologue of yeast Apg8p, is localized in autophagosome membranes after processing. EMBO J 19(21):5720-5728. https ://doi.org/10.1093/emboj/19.21.5720

9. Bjorkoy G, Lamark T, Johansen T (2006) p62/SQSTM1: a missing link between protein aggregates and the autophagy machinery. Autophagy 2(2): 138-139

10. Konecki DS, Foetisch K, Schlotter M, Lichter-Konecki U (1994) Complete cDNA sequence of human lysosome-associated membrane protein-2. Biochem Biophys Res Commun 205(1):1-5

11. Saftig P, Beertsen W, Eskelinen EL (2008) LAMP-2: a control step for phagosome and autophagosome maturation. Autophagy 4(4):510-512

12. Che H, Yan Y, Kang XH, Guo F, Yan ML, Liu HL, Hou X, Liu T, Zong DK, Sun LL, Bao YN, Sun LH, Yang BF, Ai J (2017) MicroRNA-27a promotes inefficient lysosomal clearance in the hippocampi of rats following chronic brain hypoperfusion. Mol Neurobiol 54(4):2595-2610. https://doi.org/10.1007/s1203 5-016-9856-8

13. Ben-Ari Z, Mor E, Azarov D, Sulkes J, Tor R, Cheporko Y, Hochhauser E, Pappo O (2005) Cathepsin B inactivation attenuates the apoptotic injury induced by ischemia/reperfusion of mouse liver. Apoptosis 10(6):1261-1269. https://doi.org/10.1007/s1049 5-005-2358-1

14. Xu M, Yang L, Rong JG, Ni Y, Gu WW, Luo Y, Ishidoh K, Katunuma N, Li ZS, Zhang HL (2014) Inhibition of cysteine cathepsin $\mathrm{B}$ and $\mathrm{L}$ activation in astrocytes contributes to neuroprotection against cerebral ischemia via blocking the tBid-mitochondrial apoptotic signaling pathway. Glia 62(6):855-880. https://doi. org/10.1002/glia.22645

15. Jiang Y, Woosley AN, Sivalingam N, Natarajan S, Howe PH (2016) Cathepsin-B-mediated cleavage of disabled-2 regulates TGF-beta-induced autophagy. Nat Cell Biol 18(8):851-863. https ://doi.org/10.1038/ncb3388

16. Settembre C, Di Malta C, Polito VA, Garcia Arencibia M, Vetrini F, Erdin S, Erdin SU, Huynh T, Medina D, Colella P, Sardiello M, Rubinsztein DC, Ballabio A (2011) TFEB links autophagy to lysosomal biogenesis. Science 332(6036):1429-1433. https://doi. org/10.1126/science. 1204592

17. Medina DL, Fraldi A, Bouche V, Annunziata F, Mansueto G, Spampanato C, Puri C, Pignata A, Martina JA, Sardiello M,
Palmieri M, Polishchuk R, Puertollano R, Ballabio A (2011) Transcriptional activation of lysosomal exocytosis promotes cellular clearance. Dev Cell 21(3):421-430. https://doi.org/10.1016/j. devcel.2011.07.016

18. Palmieri M, Impey S, Kang H, di Ronza A, Pelz C, Sardiello M, Ballabio A (2011) Characterization of the CLEAR network reveals an integrated control of cellular clearance pathways. Hum Mol Genet 20(19):3852-3866. https://doi.org/10.1093/hmg/ddr30 6

19. Settembre C, Zoncu R, Medina DL, Vetrini F, Erdin S, Erdin S, Huynh T, Ferron M, Karsenty G, Vellard MC, Facchinetti V, Sabatini DM, Ballabio A (2012) A lysosome-to-nucleus signalling mechanism senses and regulates the lysosome via mTOR and TFEB. EMBO J 31(5):1095-1108. https://doi.org/10.1038/emboj .2012 .32

20. Su J, Zhang T, Wang K, Zhu T, Li X (2014) Autophagy activation contributes to the neuroprotection of remote ischemic perconditioning against focal cerebral ischemia in rats. Neurochem Res 39(11):2068-2077. https://doi.org/10.1007/s11064-014-1396-x

21. Fan YY, Hu WW, Nan F, Chen Z (2017) Postconditioninginduced neuroprotection, mechanisms and applications in cerebral ischemia. Neurochem Int 107:43-56. https://doi.org/10.1016/j. neuint.2017.01.006

22. Gao L, Jiang T, Guo J, Liu Y, Cui G, Gu L, Su L, Zhang Y (2012) Inhibition of autophagy contributes to ischemic postconditioning-induced neuroprotection against focal cerebral ischemia in rats. PLoS ONE 7(9):e46092. https://doi.org/10.1371/journ al.pone. 0046092

23. Guo L, Xu JM, Mo XY (2015) Ischemic postconditioning regulates cardiomyocyte autophagic activity following ischemia/ reperfusion injury. Mol Med Rep 12(1):1169-1176. https://doi. org/10.3892/mmr.2015.3533

24. Hao M, Zhu S, Hu L, Zhu H, Wu X, Li Q (2017) Myocardial ischemic postconditioning promotes autophagy against ischemia reperfusion injury via the activation of the nNOS/AMPK/mTOR pathway. Int J Mol Sci 18 (3). https://doi.org/10.3390/ijms180306 14

25. Qi Z, Dong W, Shi W, Wang R, Zhang C, Zhao Y, Ji X, Liu KJ, Luo Y (2015) Bcl-2 phosphorylation triggers autophagy switch and reduces mitochondrial damage in limb remote ischemic conditioned rats after ischemic stroke. Transl Stroke Res 6(3):198-206. https://doi.org/10.1007/s12975-015-0393-y

26. Qi ZF, Luo YM, Liu XR, Wang RL, Zhao HP, Yan F, Song ZJ, Luo M, Ji XM (2012) AKT/GSK3beta-dependent autophagy contributes to the neuroprotection of limb remote ischemic postconditioning in the transient cerebral ischemic rat model. CNS Neurosci Ther 18(12):965-973. https://doi.org/10.1111/cns.12016

27. Wang J, Han D, Sun M, Feng J (2016) A Combination of remote ischemic perconditioning and cerebral ischemic postconditioning inhibits autophagy to attenuate plasma HMGB1 and induce neuroprotection against stroke in rat. J Mol Neurosci 58(4):424-431. https://doi.org/10.1007/s12031-016-0724-9

28. Wei C, Li H, Han L, Zhang L, Yang X (2013) Activation of autophagy in ischemic postconditioning contributes to cardioprotective effects against ischemia/reperfusion injury in rat hearts. J Cardiovasc Pharmacol 61(5):416-422. https://doi.org/10.1097/ FJC.0b013e318287d501

29. Zhang X, Yuan Y, Jiang L, Zhang J, Gao J, Shen Z, Zheng Y, Deng T, Yan H, Li W, Hou WW, Lu J, Shen Y, Dai H, Hu WW, Zhang Z, Chen Z (2014) Endoplasmic reticulum stress induced by tunicamycin and thapsigargin protects against transient ischemic brain injury: Involvement of PARK2-dependent mitophagy. Autophagy 10(10):1801-1813. https://doi.org/10.4161/auto.32136

30. Yan H, Zhang X, Hu W, Ma J, Hou W, Zhang X, Wang X, Gao J, Shen Y, Lv J, Ohtsu H, Han F, Wang G, Chen Z (2014) Histamine $\mathrm{H} 3$ receptors aggravate cerebral ischaemic injury by 
histamine-independent mechanisms. Nat Commun 5:3334. https ://doi.org/10.1038/ncomms4334

31. Zhang X, Yan H, Yuan Y, Gao J, Shen Z, Cheng Y, Shen Y, Wang RR, Wang X, Hu WW, Wang G, Chen Z (2013) Cerebral ischemia-reperfusion-induced autophagy protects against neuronal injury by mitochondrial clearance. Autophagy 9(9):1321-1333. https://doi.org/10.4161/auto.25132

32. Xie R, Wang P, Cheng M, Sapolsky R, Ji X, Zhao H (2014) Mammalian target of rapamycin cell signaling pathway contributes to the protective effects of ischemic postconditioning against stroke. Stroke 45(9):2769-2776. https://doi.org/10.1161/STROK EAHA.114.005406

33. Galluzzi L, Bravo-San Pedro JM, Blomgren K, Kroemer G (2016) Autophagy in acute brain injury. Nat Rev Neurosci 17(8):467484. https://doi.org/10.1038/nrn.2016.51

34. Baek SH, Noh AR, Kim KA, Akram M, Shin YJ, Kim ES, Yu SW, Majid A, Bae ON (2014) Modulation of mitochondrial function and autophagy mediates carnosine neuroprotection against ischemic brain damage. Stroke 45(8):2438-2443. https://doi. org/10.1161/STROKEAHA.114.005183

35. Pignataro G, Meller R, Inoue K, Ordonez AN, Ashley MD, Xiong $\mathrm{Z}$, Gala R, Simon RP (2008) In vivo and in vitro characterization of a novel neuroprotective strategy for stroke: ischemic postconditioning. J Cereb Blood Flow Metab 28(2):232-241. https://doi. org/10.1038/sj.jcbfm.9600559

36. Zhang Y, Gao L, Cheng Z, Cai J, Niu Y, Meng W, Zhao Q (2017) Kukoamine a prevents radiation-induced neuroinflammation and preserves hippocampal neurogenesis in rats by inhibiting activation of NF-kappaB and AP-1. Neurotox Res 31(2):259-268. https ://doi.org/10.1007/s12640-016-9679-4

37. Yang Y, Wang H, Li L, Li X, Wang Q, Ding H, Wang X, Ye Z, Wu L, Zhang X, Zhou M, Pan H (2016) Sinomenine provides neuroprotection in model of traumatic brain injury via the Nrf2ARE pathway. Front Neurosci 10:580. https://doi.org/10.3389/ fnins.2016.00580

38. Li X, Wang H, Gao Y, Li L, Tang C, Wen G, Zhou Y, Zhou M, Mao L, Fan Y (2016) Protective effects of quercetin on mitochondrial biogenesis in experimental traumatic brain injury via the Nrf2 signaling pathway. PLoS ONE 11(10):e0164237. https ://doi.org/10.1371/journal.pone.0164237

39. Li X, Wang H, Gao Y, Li L, Tang C, Wen G, Yang Y, Zhuang Z, Zhou M, Mao L, Fan Y (2016) Quercetin induces mitochondrial biogenesis in experimental traumatic brain injury via the PGC1alpha signaling pathway. Am J Transl Res 8(8):3558-3566

40. Zhang X, Chen S, Song L, Tang Y, Shen Y, Jia L, Le W (2014) MTOR-independent, autophagic enhancer trehalose prolongs motor neuron survival and ameliorates the autophagic flux defect in a mouse model of amyotrophic lateral sclerosis. Autophagy 10(4):588-602. https://doi.org/10.4161/auto.27710

41. Sesso A, Belizario JE, Marques MM, Higuchi ML, Schumacher RI, Colquhoun A, Ito E, Kawakami J (2012) Mitochondrial swelling and incipient outer membrane rupture in preapoptotic and apoptotic cells. Anat Rec (Hoboken) 295(10):1647-1659. https ://doi.org/10.1002/ar.22553

42. Wakabayashi T (1999) Structural changes of mitochondria related to apoptosis: swelling and megamitochondria formation. Acta Biochim Pol 46(2):223-237
43. Liu Y, Tang G, Li Y, Wang Y, Chen X, Gu X, Zhang Z, Wang Y, Yang GY (2014) Metformin attenuates blood-brain barrier disruption in mice following middle cerebral artery occlusion. J Neuroinflammation 11:177. https://doi.org/10.1186/s12974-014-0177-4

44. Zhao ZQ, Corvera JS, Halkos ME, Kerendi F, Wang NP, Guyton RA, Vinten-Johansen J (2003) Inhibition of myocardial injury by ischemic postconditioning during reperfusion: comparison with ischemic preconditioning. Am J Physiol Heart Circ Physiol 285(2):H579-H588. https://doi.org/10.1152/ajpheart.01064.2002

45. Iwai-Kanai E, Yuan H, Huang C, Sayen MR, Perry-Garza CN, Kim L, Gottlieb RA (2008) A method to measure cardiac autophagic flux in vivo. Autophagy 4(3):322-329

46. Ni HM, Bockus A, Wozniak AL, Jones K, Weinman S, Yin XM, Ding WX (2011) Dissecting the dynamic turnover of GFP-LC3 in the autolysosome. Autophagy 7(2):188-204

47. Komatsu M, Ichimura Y (2010) Physiological significance of selective degradation of p62 by autophagy. FEBS Lett 584(7):1374-1378. https://doi.org/10.1016/j.febslet.2010.02.017

48. Marwick C (1993) 'Desperate use' gene therapy guidelines ready. JAMA 269(7):843

49. He C, Klionsky DJ (2009) Regulation mechanisms and signaling pathways of autophagy. Annu Rev Genet 43:67-93. https://doi. org/10.1146/annurev-genet-102808-114910

50. Puyal J, Vaslin A, Mottier V, Clarke PG (2009) Postischemic treatment of neonatal cerebral ischemia should target autophagy. Ann Neurol 66(3):378-389. https://doi.org/10.1002/ana.21714

51. Wang JY, Xia Q, Chu KT, Pan J, Sun LN, Zeng B, Zhu YJ, Wang Q, Wang K, Luo BY (2011) Severe global cerebral ischemiainduced programmed necrosis of hippocampal CA1 neurons in rat is prevented by 3-methyladenine: a widely used inhibitor of autophagy. J Neuropathol Exp Neurol 70(4):314-322. https://doi. org/10.1097/NEN.0b013e31821352bd

52. Emanuel R, Sergin I, Bhattacharya S, Turner J, Epelman S, Settembre C, Diwan A, Ballabio A, Razani B (2014) Induction of lysosomal biogenesis in atherosclerotic macrophages can rescue lipid-induced lysosomal dysfunction and downstream sequelae. Arterioscler Thromb Vasc Biol 34(9):1942-1952. https://doi. org/10.1161/ATVBAHA.114.303342

53. Martini-Stoica H, Xu Y, Ballabio A, Zheng H (2016) The autophagy-lysosomal pathway in neurodegeneration: a TFEB perspective. Trends Neurosci 39(4):221-234. https://doi. org/10.1016/j.tins.2016.02.002

54. Tabas I (2010) Macrophage death and defective inflammation resolution in atherosclerosis. Nat Rev Immunol 10(1):36-46. https ://doi.org/10.1038/nri2675

55. Settembre C, De Cegli R, Mansueto G, Saha PK, Vetrini F, Visvikis O, Huynh T, Carissimo A, Palmer D, Klisch TJ, Wollenberg AC, Di Bernardo D, Chan L, Irazoqui JE, Ballabio A (2013) TFEB controls cellular lipid metabolism through a starvationinduced autoregulatory loop. Nat Cell Biol 15(6):647-658. https ://doi.org/10.1038/ncb2718

56. Wang X, Li L, Niu X, Dang X, Li P, Qu L, Bi X, Gao Y, Hu Y, Li M, Qiao W, Peng Z, Pan L (2014) mTOR enhances foam cell formation by suppressing the autophagy pathway. DNA Cell Biol 33(4):198-204. https://doi.org/10.1089/dna.2013.2164 